\title{
A Direct Synthesis of Bipyrroles Using Phenyliodine Bis(trifluoroacetate) with
}

\section{Bromotrimethylsilane}

Toshifumi Dohi, Koji Morimoto, Akinobu Maruyama, and Yasuyuki Kita*

Graduate Scool of Pharmaceutical Sciences, Osaka University, 1-6 Yamada-oka, Suita, Osaka 565-0871, Japan

\section{General Information}

${ }^{1} \mathrm{H}$ NMR and ${ }^{13} \mathrm{C}$ NMR spectra were recorded on a JEOL JMN-300 spectrometer in $\mathrm{CDCl}_{3}$ with tetramethylsilane as an internal standard. Data are reported as follows: chemical shift in ppm $(\delta)$, multiplicity $(\mathrm{s}=$ singlet, $\mathrm{d}=$ doublet, $\mathrm{t}=$ triplet, $\mathrm{q}=$ quartet, br $=$ broad singlet, $\mathrm{m}=$ multiplet), coupling constant $(\mathrm{Hz})$, integration, and interpretation. Infrared spectra (IR) were obtained on a Hitachi 270-50 spectrometer; absorptions are reported in reciprocal centimeters with the following relative intensities: $\mathrm{s}$ (strong), m (medium), or w (weak). Mass spectra were obtained on a Shimadzu GCMS-QP 5000 instrument with ionization voltages of $70 \mathrm{eV}$. High resolution mass spectra and Elemental analyses were performed by the Elemental Analysis Section of Osaka University. Column chromatography was performed with $\mathrm{SiO}_{2}$ (Merck Silica Gel $60(230-400$ mesh)).

\section{Materials}

PIFA was prepared from commercially available phenyliodine diacetate (PIDA) and trifluoroacetic acid. ${ }^{1}$ Absolute dichloromethane, TMSBr and pyrroles $(\mathbf{3 b}, \mathbf{3 e}, \mathbf{f}, \mathbf{8})$ are commercially available and used without further purification. Other $1 H$-pyrroles (3a and $\mathbf{c},{ }^{2} \mathbf{3 d},{ }^{3} \mathbf{3 f},{ }^{4} \mathbf{3 h}-\mathbf{j},{ }^{5} \mathbf{3} \mathbf{k}^{6}$ ) and indole $\mathbf{5}^{7}$ were prepared according to the literature procedure.

4-(1H-Pyrrol-3-yl)butyric acid methyl ester (3h): Colorless oil; $R_{f}=0.52$ (hexane/EtOAc = 2/1); ${ }^{1} \mathrm{H} \mathrm{NMR}\left(\mathrm{CDCl}_{3}\right): 1.91(\mathrm{~m}, 2 \mathrm{H}), 2.35(\mathrm{t}, J=7.8 \mathrm{~Hz}, 2 \mathrm{H}), 2.53$ 
(t, $J=7.8 \mathrm{~Hz}, 2 \mathrm{H}), 3.66(\mathrm{~s}, 3 \mathrm{H}), 6.08(\mathrm{~m}, 1 \mathrm{H}), 6.56(\mathrm{~m}, 1 \mathrm{H}), 6.70(\mathrm{~m}, 1 \mathrm{H}), 8.16(\mathrm{bs}$, $1 \mathrm{H}) ;{ }^{13} \mathrm{C} \mathrm{NMR}\left(\mathrm{CDCl}_{3}\right): \delta 26.19,26.25,33.57,51.38,108.35,115.13,117.73,122.90$, 174.37; IR (KBr): 3396 w, 2949 m, 2853 w, 1728 s, 1439 m, 1261 s, 1144 m, 1065 m, $1001 \mathrm{w}, 912 \mathrm{~m}, 889 \mathrm{w}, 748 \mathrm{~s} \mathrm{~cm}^{-1}$; HRFABMS Calcd for $\mathrm{C}_{9} \mathrm{H}_{14} \mathrm{NO}_{2}(\mathrm{M}+\mathrm{H})^{+} ; 168.1025$. Found 168.1014.

\section{General Procedure for PIFA-mediated Oxidative Coupling Reaction of 1H-Pyrroles}

\section{Leading to 2, 2'-Bipyrroles}

To a stirred solution of $1 H$-pyrrole 3a (111 mg, $0.9 \mathrm{mmol})$ in $\mathrm{CH}_{2} \mathrm{Cl}_{2}(15 \mathrm{~mL})$, PIFA $(0.3 \mathrm{mmol})$ and $\mathrm{TMSBr}(0.6 \mathrm{mmol})$ were quickly added at $-78^{\circ} \mathrm{C}$. The reaction mixture was then stirred for 1 hour, while the reaction temperature was maintained below $-40^{\circ} \mathrm{C}$. After the reaction completion, saturated aqueous $\mathrm{NaHCO}_{3}$ (ca. $20 \mathrm{~mL}$ ) was added to the mixture, and then stirred for an additional 10 minutes at ambient temperature. The organic layer was separated and the aqueous phase was extracted with $\mathrm{CH}_{2} \mathrm{Cl}_{2}$. The combined extract was dried with $\mathrm{Na}_{2} \mathrm{SO}_{4}$ and evaporated to dryness. The residue was purified by column chromatography $\left(\mathrm{SiO}_{2}\right.$ (neutral) $/ n$-hexane-AcOEt) to give the pure 2 , 2'-bipyrrole 4a (56 mg, 75\%) as a colorless oil. The less polar fractions gave unreacted 3a as a pure form $(31 \mathrm{mg})$.

Note: All 1H, 1'H-2, 2'-bipyrroles (4) are not air-stable and decompose within 1 month at room temperature, thus should be stored in refrigerator under an atmosphere of nitrogen.

3, 4, 3', 4'-Tetraethyl-1H, 1'H-[2, 2']bipyrrolyl (4a) ${ }^{8}$ : white powder; m.p. 90-92 ${ }^{\circ} \mathrm{C}$, $R_{f}=0.35($ hexane/EtOAc $=4 / 1) ;{ }^{1} \mathrm{H} \mathrm{NMR}\left(\mathrm{CDCl}_{3}\right): 1.05(\mathrm{t}, J=7.8 \mathrm{~Hz}, 4 \mathrm{H}), 1.25(\mathrm{t}, J=$ $7.8 \mathrm{~Hz}, 4 \mathrm{H}), 2.42$ (q, $J=7.8 \mathrm{~Hz}, 4 \mathrm{H}), 2.52$ (q, $J=7.8 \mathrm{~Hz}, 4 \mathrm{H}), 6.53$ (bs, 2H), 7.58 (bs, $2 \mathrm{H}) ;{ }^{13} \mathrm{C}$ NMR $\left(\mathrm{CDCl}_{3}\right): 14.33,16.02,17.93,18.59,113.84,121.09,122.88,125.15$; IR (KBr): 3373 m, 2963 s, 2867 m, 1553 w, 1435 m, 1371 m, 1327 w, 1259 w, 1190 w, $1086 \mathrm{~m}, 1063 \mathrm{~m}, 1038 \mathrm{~m}, 947 \mathrm{~m}, 912 \mathrm{~s}, 742 \mathrm{~s}, 650 \mathrm{~m} \mathrm{~cm}^{-1}$; MS, m/z: $244\left(\mathrm{M}^{+}\right)$. 
1H, 1'H-[2, 2']Bipyrrolyl (4b) ${ }^{9}$ : white powder; m.p. 187-189 ${ }^{\circ} \mathrm{C}, R_{f}=0.32$ (hexane/EtOAc $=4 / 1) ;{ }^{1} \mathrm{H}-\mathrm{NMR}\left(\mathrm{CDCl}_{3}\right): \delta$ 6.05-6.19 $(\mathrm{m}, 4 \mathrm{H}), 6.70-6.72(\mathrm{~m}, 2 \mathrm{H}), 8.23$ (bs, $2 \mathrm{H}) ;{ }^{13} \mathrm{C} \mathrm{NMR}\left(\mathrm{CDCl}_{3}\right): \delta 103.5,109.4,117.6,125.9$; IR (KBr): 3366 m, $3123 \mathrm{w}$, 3103 w, 1574 w, 1518 w, 1454 w, 1425 w, 1404 w, 1261 w, 1097 m, 1032 m, 912 s, 891 w, $775 \mathrm{~m}, 743 \mathrm{~s}, 658 \mathrm{w} \mathrm{cm}^{-1}$; MS, $m / z: 132\left(\mathrm{M}^{+}\right)$.

3, 4, 3', 4'-Tetraisobutyl-1H, 1'H-[2, 2']bipyrrolyl (4c): light yellow oil; $R_{f}=0.31$ (hexane/ $\left.\mathrm{CH}_{2} \mathrm{Cl}_{2}=2 / 1\right) ;{ }^{1} \mathrm{H} \mathrm{NMR}\left(\mathrm{CDCl}_{3}\right): \delta 0.78(\mathrm{~d}, J=6.6 \mathrm{~Hz}, 12 \mathrm{H}), 0.93(\mathrm{~d}, J=6.6$ $\mathrm{Hz}, 12 \mathrm{H}), 1.64(\mathrm{~m}, 2 \mathrm{H}), 1.80(\mathrm{~m}, 2 \mathrm{H}), 2.23$ (d, J=7.2 Hz, 4H), 2.30 (d, J=7.2 Hz, 4H), $6.48(\mathrm{~d}, J=2.4 \mathrm{~Hz}, 2 \mathrm{H}), 7.66(\mathrm{bs}, 2 \mathrm{H}) ;{ }^{13} \mathrm{C} \mathrm{NMR}\left(\mathrm{CDCl}_{3}\right): \delta 22.64,22.74,29.22,29.30$, 34.27, 35.14, 114.62, 120.48, 121.97, 122.73; IR (KBr): 3477 m, 3379 m, 2866 m, 1464 m, 1433 m, 1381 w, 1364 w, 1339 w, 1167 w, 1091 w, 912 s, 743 s, $650 \mathrm{~m} \mathrm{~cm}^{-1}$; HRFABMS Calcd for $\mathrm{C}_{24} \mathrm{H}_{40} \mathrm{~N}_{2} ; 356.3691$. Found 356.3686.

4, 5, 6, 7, 4', 5', 6', 7'-Octahydro-2H, 2' $H$-[1, 1']biisoindolyl (4d): light yellow oil ; $R_{f}$ $=0.32($ hexane $/$ EtOAc $=4 / 1) ;{ }^{1} \mathrm{H}$ NMR $\left(\mathrm{CD}_{2} \mathrm{Cl}_{2}\right): \delta 1.75(\mathrm{~m}, 8 \mathrm{H}), 2.53-2.68(\mathrm{~m}, 8 \mathrm{H})$, 6.46 (bs, 2H), 7.92 (bs, 2H); ${ }^{13} \mathrm{C}$ NMR $\left(\mathrm{CDCl}_{3}\right): \delta 21.83,22.59,23.54,23.87,112.01$, 114.73, 120.07, 120.23; IR (KBr): 3442 s, 3371 m, 2930 s, 2847 m, 1560 m, 1435 s, 1387 w, 1317 w, 1256 m, 1184 m, 1132 m, 1076 m, 1032 m, 955 s, 916 m, 822 w, 733 s, $650 \mathrm{w} \mathrm{cm}^{-1}$; HRFABMS Calcd for $\mathrm{C}_{16} \mathrm{H}_{20} \mathrm{~N}_{2} ; 240.1626$. Found 240.1641.

In the reaction of 3 -substituted $1 H$-pyrroles $(\mathbf{3 e}-\mathbf{j})$, two regioisomers (head to tail dimer (4'e-j, major product) and head to head dimer (4')e-j, minor product)) were formed and separatable by usual column chromatography techniques $\left(\mathrm{SiO}_{2}\right.$ (neutral) $/ n$-hexane-AcOEt). The regiochemistry of the products was determined according to the literature. ${ }^{10}$

3, 4'-Dimethyl-1H, 1'H-[2, 2']bipyrrolyl (4'e, major) ${ }^{10}$ : light yellow oil; $R_{f}=0.31$ (hexane/EtOAc $=4 / 1) ;{ }^{1} \mathrm{H}$ NMR $\left(\mathrm{CDCl}_{3}\right): 2.14(\mathrm{~s}, 3 \mathrm{H}), 2.20(\mathrm{~s}, 3 \mathrm{H}), 6.00-6.04(\mathrm{~m}, 1 \mathrm{H})$, $6.07(\mathrm{t}, J=2.4 \mathrm{~Hz}, 1 \mathrm{H}), 6.49-6.53(\mathrm{~m}, 1 \mathrm{H}), 6.59(\mathrm{t}, J=2.4 \mathrm{~Hz}, 1 \mathrm{H}), 7.93(\mathrm{bs}, 2 \mathrm{H}) ;{ }^{13} \mathrm{C}$ 
$\operatorname{NMR}\left(\mathrm{CD}_{2} \mathrm{Cl}_{3}\right): 11.84,11.97,106.72,111.27,114.49,115.39,116.53,119.62,122.07$, 125.64; IR (KBr): 3377 m, 3096 w, 2924 m, 2866 m, 1562 m, 1504 m, 1435 m, 1381 m, 1261 m, 1207 w, 1173 m, 1097 s, 1063 m, 1030 m, 972 m, 912 s, 889 s, 793 s, 741 s, $689 \mathrm{~m} \mathrm{~cm}^{-1}$; MS, $m / z: 160\left(\mathrm{M}^{+}\right)$.

3, 3'-Dimethyl-1H, 1'H-[2, 2']bipyrrolyl (4"e, minor) ${ }^{10}$ : light yellow oil; $R_{f}=0.35$ (hexane/EtOAc $=4 / 1) ;{ }^{1} \mathrm{H}$ NMR $\left(\mathrm{CD}_{2} \mathrm{Cl}_{2}\right): \delta 2.16(\mathrm{~s}, 6 \mathrm{H}), 6.14(\mathrm{t}, J=2.4 \mathrm{~Hz}, 2 \mathrm{H}), 6.72$ (t, $J=2.4 \mathrm{~Hz}, 2 \mathrm{H}), 7.95(\mathrm{bs}, 2 \mathrm{H})$; MS, $m / z: 160\left(\mathrm{M}^{+}\right)$.

3, 4'-Diheptyl-1H, 1'H-[2, 2']bipyrrolyl (4'f, major): light yellow oil; $R_{f}=0.30$ (hexane/EtOAc $=4 / 1) ;{ }^{1} \mathrm{H}$ NMR $\left(\mathrm{CDCl}_{3}\right): 0.80-0.95(\mathrm{~m}, 6 \mathrm{H}), 1.22-1.38(\mathrm{~m}, 20 \mathrm{H})$, 1.53-1.64 (m, 4H), $2.48(\mathrm{t}, J=7.5 \mathrm{~Hz}, 2 \mathrm{H}), 2.56(\mathrm{t}, J=7.5 \mathrm{~Hz}, 2 \mathrm{H}), 6.04(\mathrm{~m}, 1 \mathrm{H}), 6.12$ (t, $J=2.7 \mathrm{~Hz}, 1 \mathrm{H}), 6.56(\mathrm{~m}, 1 \mathrm{H}), 6.68(\mathrm{t}, J=2.7 \mathrm{~Hz}, 1 \mathrm{H}), 7.91(\mathrm{bs}, 1 \mathrm{H}), 7.95(\mathrm{bs}, 1 \mathrm{H})$;

${ }^{13} \mathrm{C} \mathrm{NMR}\left(\mathrm{CDCl}_{3}\right)$ : 14.11, 22.67, 26.49, 27.00, 29.24, 29.52, 29.62, 31.06, 31.18, 31.89, 31.90, 106.03, 109.78, 114.59, 116.57, 120.43, 121.71, 125.41, 125.59; IR (KBr): 3371 w, 2924 s, 2853 m, 1684 w, 1437 w, 1377 w, 1107 w, 976 w, 891 w cm'- HRFABMS Calcd for $\mathrm{C}_{22} \mathrm{H}_{36} \mathrm{~N}_{2} ; 328.2878$. Found 328.2862.

3, 3'-Diheptyl-1H, 1'H-[2, 2']bipyrrolyl (4'f, minor): light yellow oil; $R_{f}=0.34$ (hexane/EtOAc $=4 / 1) ;{ }^{1} \mathrm{H}$ NMR $\left(\mathrm{CD}_{2} \mathrm{Cl}_{2}\right): \delta$ 0.80-0.92 (m, 6H), 1.22-1.30 (m, 20H), 1.57-1.55 (m, 4H), 2.39 (t, $J=7.8 \mathrm{~Hz}, 4 \mathrm{H}), 6.12(\mathrm{t}, J=2.7 \mathrm{~Hz}, 2 \mathrm{H}), 6.74$ (t, J = 2.7 Hz, 2H), 7.97 (bs, 2H); MS, $m / z: 328\left(\mathrm{M}^{+}\right)$.

\section{5-[3'-(3-Methoxycarbonylpropyl)-1H, 1'H-[2, 2']bipyrrolyl-3-yl]butanoic acid}

methyl ester (4'g, major): light yellow oil; $R_{f}=0.33$ (hexane/EtOAc $\left.=2 / 1\right) ;{ }^{1} \mathrm{H} \mathrm{NMR}$ $\left(\mathrm{CDCl}_{3}\right): 1.82-2.03(\mathrm{~m}, 4 \mathrm{H}), 2.33-2.46(\mathrm{~m}, 4 \mathrm{H}), 2.54(\mathrm{t}, J=7.8 \mathrm{~Hz}, 2 \mathrm{H}), 2.61(\mathrm{t}, J=7.8$ $\mathrm{Hz}, 2 \mathrm{H}), 3.67$ (s, 3H), $3.70(\mathrm{~s}, 3 \mathrm{H}), 6.03(\mathrm{~m}, 1 \mathrm{H}), 6.07$ (t, $J=2.7 \mathrm{~Hz}, 1 \mathrm{H}), 6.63(\mathrm{~m}, 1 \mathrm{H})$, $6.67(\mathrm{t}, J=2.7 \mathrm{~Hz}, 1 \mathrm{H}), 8.09(\mathrm{bs}, 1 \mathrm{H}), 9.03$ (bs, $1 \mathrm{H}) ;{ }^{13} \mathrm{C}$ NMR $\left(\mathrm{CDCl}_{3}\right)$ : $\delta 25.72,26.19,26.30,26.37,32.87,33.61,51.46,51.72,104.93,109.92,115.42,116.57$ , 118.30, 122.29, 123.60, 125.43, 174.42, 175.16; IR (KBr): 3377 m, 2949 m, 2853 m, 1713 s, 1556 m, 1537 w, 1504 m, 1454 m, 1433 m, 1140 m, 1101 w, 1003 w, 912 s, 797 
w, $743 \mathrm{~s} \mathrm{~cm}^{-1}$. HRFABMS Calcd for $\mathrm{C}_{18} \mathrm{H}_{24} \mathrm{~N}_{2} \mathrm{O}_{4} \mathrm{Na}(\mathrm{M}+\mathrm{Na})^{+} ; 355.1634$ Found 355.1628 .

\section{4-[3'-(3-Methoxycarbonylpropyl)-1H, 1'H-[2, 2']bipyrrolyl-3-yl]butyric acid}

methyl ester (4"'g, minor): light yellow oil; $R_{f}=0.36($ hexane/EtOAc $=2 / 1) ;{ }^{1} \mathrm{H} \mathrm{NMR}$ $\left(\mathrm{CDCl}_{3}\right): 1.82-1.99(\mathrm{~m}, 4 \mathrm{H}), 2.35(\mathrm{t}, J=7.3 \mathrm{~Hz}, 4 \mathrm{H}), 2.52(\mathrm{t}, J=7.3 \mathrm{~Hz}, 4 \mathrm{H}), 3.64(\mathrm{~s}$, $6 \mathrm{H}), 6.12(\mathrm{t}, J=2.7 \mathrm{~Hz}, 2 \mathrm{H}), 6.78(\mathrm{t}, J=2.7 \mathrm{~Hz}, 2 \mathrm{H}), 8.61(\mathrm{bs}, 2 \mathrm{H}) ; \mathrm{MS}, \mathrm{m} / \mathrm{z}: 332$ $\left(\mathrm{M}^{+}\right)$.

3, 4'-Diphenyl-1H, 1'H-[2, 2']bipyrrolyl (4'h, major): light yellow oil; $R_{f}=0.28$ (hexane/ $\left.\mathrm{CH}_{2} \mathrm{Cl}_{2}=1 / 1\right) ;{ }^{1} \mathrm{H} \mathrm{NMR}\left(\mathrm{CDCl}_{3}\right): \delta 6.35(\mathrm{t}, J=2.7 \mathrm{~Hz}, 1 \mathrm{H}), 6.51(\mathrm{~m}, 1 \mathrm{H}), 6.74$ $(\mathrm{t}, J=2.7 \mathrm{~Hz}, 1 \mathrm{H}), 6.86(\mathrm{~m}, 1 \mathrm{H}), 7.09-7.51(\mathrm{~m}, 10 \mathrm{H}), 7.98(\mathrm{bs}, 1 \mathrm{H}), 8.14(\mathrm{bs}, 1 \mathrm{H}) ;{ }^{13} \mathrm{C}$ NMR $\left(\mathrm{CDCl}_{3}\right): \delta 103.45,109.83,110.21,114.63,117.89,120.80,120.98,124.91$, 125.23, 125.60, 125.82, 126.05, 128.08, 128.64, 135.25, 136.18; IR (KBr): 3379 w, 2855 w, 1790 w, 1682 w, 1177 m, 912 s, 743 s, $650 \mathrm{~m} \mathrm{~cm}^{-1}$; HRFABMS Calcd for $\mathrm{C}_{14} \mathrm{H}_{16} \mathrm{~N}_{2} ; 284.1313$. Found 284.1299.

3, 3'-Diphenyl-1H, 1'H-[2, 2']bipyrrolyl (4''h, minor) : light yellow oil; $R_{f}=0.34$ (hexane $\left./ \mathrm{CH}_{2} \mathrm{Cl}_{2}=1 / 1\right) ;{ }^{1} \mathrm{H} \mathrm{NMR}\left(\mathrm{CDCl}_{3}\right): \delta 6.46(\mathrm{t}, J=2.7 \mathrm{~Hz}, 2 \mathrm{H}), 6.74(\mathrm{t}, J=2.7$ $\mathrm{Hz}, 2 \mathrm{H}), 7.14-7.22(\mathrm{~m}, 2 \mathrm{H}), 7.23-7.36(\mathrm{~m}, 4 \mathrm{H}), 7.38-7.45$ (m, 4H), 8.00 (bs, 2H); MS, $m / z: 284\left(\mathrm{M}^{+}\right)$.

3, 4'-Bis(4-methoxyphenyl)-1H, 1'H-[2, 2']bipyrrolyl (4'i, sole product): light yellow oil; $R_{f}=0.35($ hexane/EtOAc $=2 / 1) ;{ }^{1} \mathrm{H}$ NMR $\left(\mathrm{CDCl}_{3}\right): \delta 3.82(\mathrm{~s}, 3 \mathrm{H}), 3.83(\mathrm{~s}, 3 \mathrm{H})$, $6.32(\mathrm{t}, J=2.7 \mathrm{~Hz}, 1 \mathrm{H}), 6.49(\mathrm{~m}, 1 \mathrm{H}), 6.81(\mathrm{t}, J=2.7 \mathrm{~Hz}, 1 \mathrm{H}), 6.85(\mathrm{~m}, 1 \mathrm{H}), 6.86-6.94$ (m, 4H), 7.35 (d, $J=8.4 \mathrm{~Hz}, 2 \mathrm{H}), 7.44$ (d, $J=8.4 \mathrm{~Hz}, 1 \mathrm{H}), 8.04$ (bs, 1H), 8.27 (bs, 1H); ${ }^{13} \mathrm{C} \mathrm{NMR}\left(\mathrm{CDCl}_{3}\right): \delta 55.26,55.29,102.92,110.36,113.67,114.08,114.11,117.56$, $120.39,120.81,125.08,126.00,126.09,128.19,128.68,129.40,157.79,158.11$; IR (KBr): 3369 m, 3002 w, 2937 w, 2909 w, 2835 w, 1611 w, 1556 w, 1526 m, 1510 m, 1493 m, 1464 m, 1441 m, 1425 w, 1391 w, 1288 m, 1244 s, 1178 m, 1109 m, 1096 w, 1076 w, 1030 m, 912 s, 833 m, 793 m, 743 s, 698 m, $650 \mathrm{~m} \mathrm{~cm}^{-1}$. HRFABMS Calcd for 
$\mathrm{C}_{22} \mathrm{H}_{20} \mathrm{~N}_{2} \mathrm{O}_{2} ; 344.1525$. Found 344.1519.

3, 4'-Bis(4-bromophenyl)-1H, 1'H-[2, 2']bipyrrolyl (4'j' major): light yellow oil; $R_{f}$ $=0.32\left(\right.$ hexane $\left./ \mathrm{CH}_{2} \mathrm{Cl}_{2}=1 / 2\right) ;{ }^{1} \mathrm{H} \mathrm{NMR}\left(\mathrm{CDCl}_{3}\right): \delta 6.36(\mathrm{t}, J=2.7 \mathrm{~Hz}, 1 \mathrm{H}), 6.53(\mathrm{~m}$, 1H), $6.83(\mathrm{t}, J=2.7 \mathrm{~Hz}, 1 \mathrm{H}), 6.96(\mathrm{~m}, 1 \mathrm{H}), 7.25-7.52(\mathrm{~m}, 8 \mathrm{H}), 8.02(\mathrm{bs}, 1 \mathrm{H}), 8.26$ (bs, $1 \mathrm{H}) ;{ }^{13} \mathrm{C}$ NMR $\left(\mathrm{CDCl}_{3}\right): \delta 103.84,110.16,114.95,118.24,119.17,119.92,119.98$, $120.94,124.52,125.69,126.53,129.64,131.69,131.76,134.14,134.97$; IR (KBr): 3450 w, 3369 w, 1591 w, 1514 m, 1494 m, 1479 m, 1441 w, 1410 w, 1385 w, 1261 w, 1175 w, 1105 m, 1070 m, 1009 m, 912 s, 829 m, 800 m, 743 s, 650 w cm. HRFABMS Calcd for $\mathrm{C}_{20} \mathrm{H}_{14} \mathrm{~N}_{2} \mathrm{Br}_{2} ; 439.9524$. Found 439.9529.

3, 3'-Bis(4-bromophenyl)-1H, 1'H-[2, 2']bipyrrolyl (4'’j, minor): light yellow oil; $R_{f}$ $=0.36\left(\right.$ hexane $\left./ \mathrm{CH}_{2} \mathrm{Cl}_{2}=1 / 2\right) ;{ }^{1} \mathrm{H} \mathrm{NMR}\left(\mathrm{CDCl}_{3}\right): \delta 6.45(\mathrm{t}, J=2.7 \mathrm{~Hz}, 2 \mathrm{H}), 6.79(\mathrm{t}, J=$ $2.7 \mathrm{~Hz}, 2 \mathrm{H}), 7.21(\mathrm{~d}, J=8.4 \mathrm{~Hz}, 4 \mathrm{H}), 7.39$ (d, $J=8.4 \mathrm{~Hz}, 4 \mathrm{H}), 8.00$ (bs, 2H); MS, $m / z$ : $439\left(\mathrm{M}^{+}\right)$.

From $1 \mathrm{H}$-indole $\mathbf{5}$, bisindole $\mathbf{6}$ produced as a major product together with the addition product 7 in a similar way for the synthesis of 2, 2'-bipyrroles.

3, 3'-Dimethyl-1H, 1'H-[2, 2']biindolyl (6) ${ }^{11}$ : white powder; m.p. $158-161{ }^{\circ} \mathrm{C} ; R_{f}=$ 0.29 (hexane $\left./ \mathrm{CH}_{2} \mathrm{Cl}_{2}=1 / 1\right) ;{ }^{1} \mathrm{H} \mathrm{NMR}\left(\mathrm{CDCl}_{3}\right): \delta 2.37(\mathrm{~s}, 6 \mathrm{H}), 7.17(\mathrm{t}, J=7.0 \mathrm{~Hz}, 2 \mathrm{H})$, $7.25(\mathrm{t}, J=7.2 \mathrm{~Hz}, 2 \mathrm{H}), 7.35$ (d, $J=8.1 \mathrm{~Hz}, 2 \mathrm{H}), 7.61$ (d, $J=7.7 \mathrm{~Hz}, 2 \mathrm{H}), 7.92$ (bs, $2 \mathrm{H}) ;{ }^{13} \mathrm{C} \mathrm{NMR}\left(\mathrm{CDCl}_{3}\right): \delta 9.74,110.70,118.85,119.58,122.49,126.61,129.13$, 136.10; IR (KBr): 3406 w, 3057 w, 2918 w, 2860 w, 1551 w, 1454 m, 1420 w, 1333 w, 1240 w, 1176 w, 1152 w, 1115 w, 1099 w, 1011 w, 912 s, 745 s, $650 \mathrm{w} \mathrm{cm}^{-1}$; MS, m/z: $260\left(\mathrm{M}^{+}\right)$.

3, 3'-Dimethyl-2, 3-dihydro-1H, $\mathbf{1}^{\prime} \boldsymbol{H}$-[2, 2']biindolyl (7) ${ }^{12}$ : white powder; m.p. 126-129 ${ }^{\circ} \mathrm{C} ; R_{f}=0.40$ (hexane/ $\left.\mathrm{CH}_{2} \mathrm{Cl}_{2}=1 / 1\right) ;{ }^{1} \mathrm{H} \mathrm{NMR}\left(\mathrm{CDCl}_{3}\right): \delta 1.35(\mathrm{~d}, J=6.6 \mathrm{~Hz}$, 3H), $2.31(\mathrm{~s}, 3 \mathrm{H}), 3.16-3.26(\mathrm{~m}, 1 \mathrm{H}), 3.92(\mathrm{bs}, 1 \mathrm{H}), 4.68(\mathrm{~d}, J=10.5 \mathrm{~Hz}, 1 \mathrm{H}), 6.65(\mathrm{~d}, J$ $=7.8 \mathrm{~Hz}, 1 \mathrm{H}), 6.81(\mathrm{t}, J=7.5 \mathrm{~Hz}, 1 \mathrm{H}), 7.02-7.18(\mathrm{~m}, 2 \mathrm{H}), 7.22(\mathrm{~d}, J=7.8 \mathrm{~Hz}, 1 \mathrm{H}), 7.52$ 
$(\mathrm{d}, J=7.5 \mathrm{~Hz}, 1 \mathrm{H}), 8.30(\mathrm{bs}, 1 \mathrm{H}) ;{ }^{13} \mathrm{C} \mathrm{NMR}\left(\mathrm{CDCl}_{3}\right): \delta 8.65,16.42,45.15,64.23$, $108.28,109.51,110.65,118.38,119.41,119.58,121.81,123.23,127.66,129.15,132.89$, 134.42, 135.17, 149.72; IR (KBr): 3353 w, 3030 w, 2862 w, 1614 w, 1485 w, 1469 w, 1452 w, 1290 w, 1203 w, 1153 w, 1084 w, 1003 w, 912 s, 743 s, $650 \mathrm{w} \mathrm{cm}^{-1}$; MS, m/z: $262\left(\mathrm{M}^{+}\right)$.

\section{Transformation of 8 into 2, 3'-bipyrrole 9}

To a stirred solution of 8 ( 3 equiv) in $\mathrm{CH}_{2} \mathrm{Cl}_{2}$, PIFA ( 1 equiv) and TMSBr (2 equiv) were quickly added at $-78^{\circ} \mathrm{C}$. The reaction mixture was then stirred for over night, while the reaction temperature was gradually warmed to ambient temperature. Saturated $\mathrm{NaHCO}_{3}$ aq. was added to the mixture, and then stirred for an additional 10 minutes. The organic layer was separated and then dried with $\mathrm{Na}_{2} \mathrm{SO}_{4}$. After evaporation, the residue was purified by column chromatography $\left(\mathrm{SiO}_{2}\right.$ (neutral) $/ n$-hexane-AcOEt) to give the 2, 3'-bipyrrole (9) (56\%) as an oil.

1, 1'-Diphenyl-1H, 1'H-[2, 3']bipyrrolyl (9) ${ }^{13}$ : light yellow oil; $R_{f}=0.27$ (hexane/EtOAc $=20 / 1) ;{ }^{1} \mathrm{H} \mathrm{NMR}\left(\mathrm{CDCl}_{3}\right): 6.07(\mathrm{dd}, J=3.3,1.7 \mathrm{~Hz}, 1 \mathrm{H}), 6.31-6.40(\mathrm{~m}$, 2H), 6.63-6.66 (m, 1H), 6.83-6.86 (m, 1H), 6.90-6.94 (m, 1H), 7.16-7.28 (m, 4H), 7.31-7.40 (m, 6H); ${ }^{13} \mathrm{C} \mathrm{NMR}\left(\mathrm{CDCl}_{3}\right): 107.85,108.73,110.50,116.16,118.35,118.82$, $119.83,122.75,125.30,126.45,126.94,128.70,128.99,129.39,140.21,140.74$; IR (KBr): 3058 w, 1599 w, 1504 m, 1462 w, 1352 w, 1319 w, 1184 w, 1074 w, 1036 w, 912 s, $743 \mathrm{~s}, 694 \mathrm{w}, 650 \mathrm{w} \mathrm{cm}{ }^{-1}$; HRFABMS Calcd for $\mathrm{C}_{20} \mathrm{H}_{17} \mathrm{~N}_{2}(\mathrm{M}+\mathrm{H})^{+} ; 285.1392$. Found 285.1394 .

\section{References}

1) White, J. D.; Caravatti, G.; Kline, T. B.; Edstrom, E.; Rice, K. C.; Brossi, A. Tetrahedron 1983, 39, 2393.

2) Franck, B.; Nonn, A.; Fuchs, K.; Gosmann, M. Liebigs Ann. Chem. 1994, 503. 
3) Finikova, O. S.; Cheprakov, A. V.; Beletskaya, I. P.; Carroll, P. J.; Vinogradov, S. A. J. Org. Chem. 2004, 69, 522.

4) Zelikin, A.; Shastri, V. R.; Langer, R. J. Org. Chem. 1999, 64, 3379.

5) Smith, N. D.; Huang, D.; Cosford, N. D. P. Org. Lett. 2002, 4, 3537.

6) Dohi, T.; Morimoto, K.; Kiyono, Y.; Tohma, H.; Kita, Y. Org. Lett. 2005, 7, 537.

7) Rossiter, S. J. Chem. Soc. 1953, 3654.

8) Shevchnk, S. V.; Davis, J. M.; Sessler, J. L. Tetrahedron Lett. 2001, 42, 2447.

9) Rapoport, C. J. Am. Chem. Soc. 1962, 84, 2178.

10) Benincori, T.; Brenna, E.; Sannicolò, F.; Zotti, G.; Zecchin, S.; Schiavon, G.; Gatti, C.; Frigerio, G. Chem. Mater. 2000, 12, 1480.

11) Benincori, T.; Brenna, E.; Sannicolò, F.; Trimarco, L.; Antognazza, P.; Cesarotti, E.; Demartin, F.; Pilati, T.; Zotti, G. J. Organomet. Chem. 1997, 529, 445.

12) Hinman, S. J. Org. Chem. 1966, 26, 2339.

13) Farnier, M.; Soth, S.; Fournari, P. Can. J. Chem. 1976, 54, 1083. 

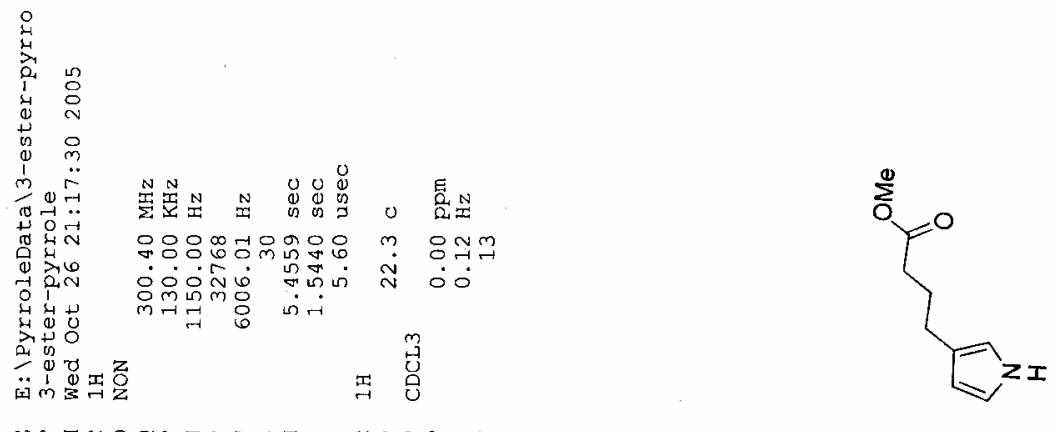

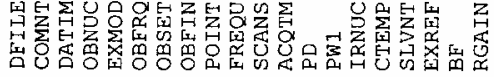

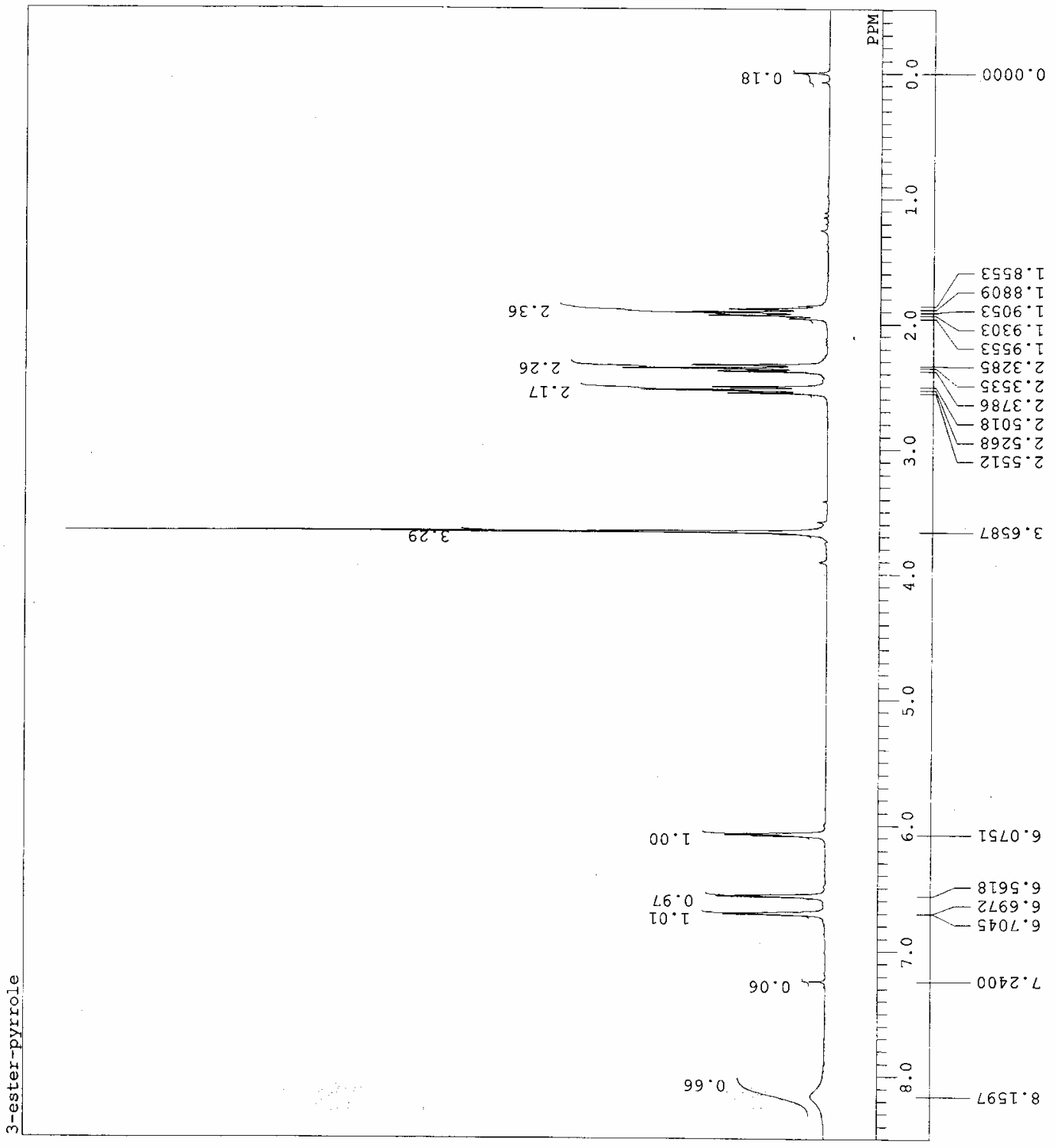



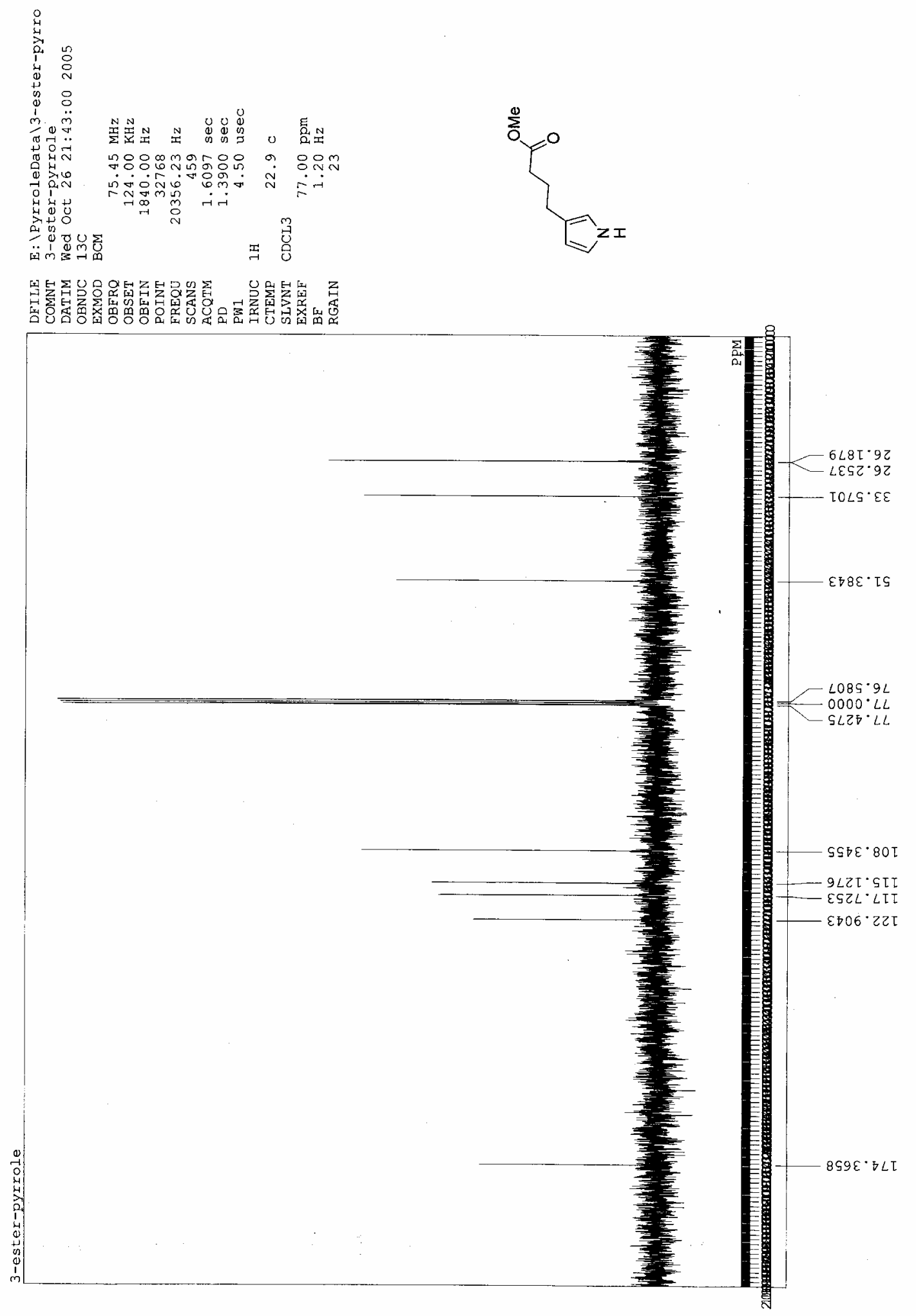

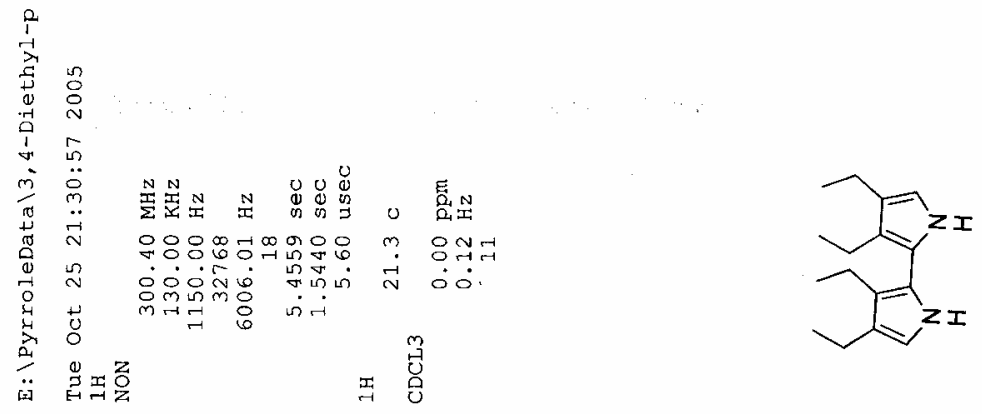

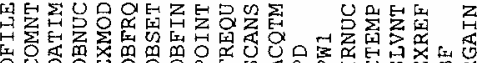

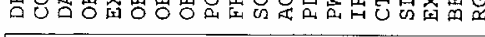

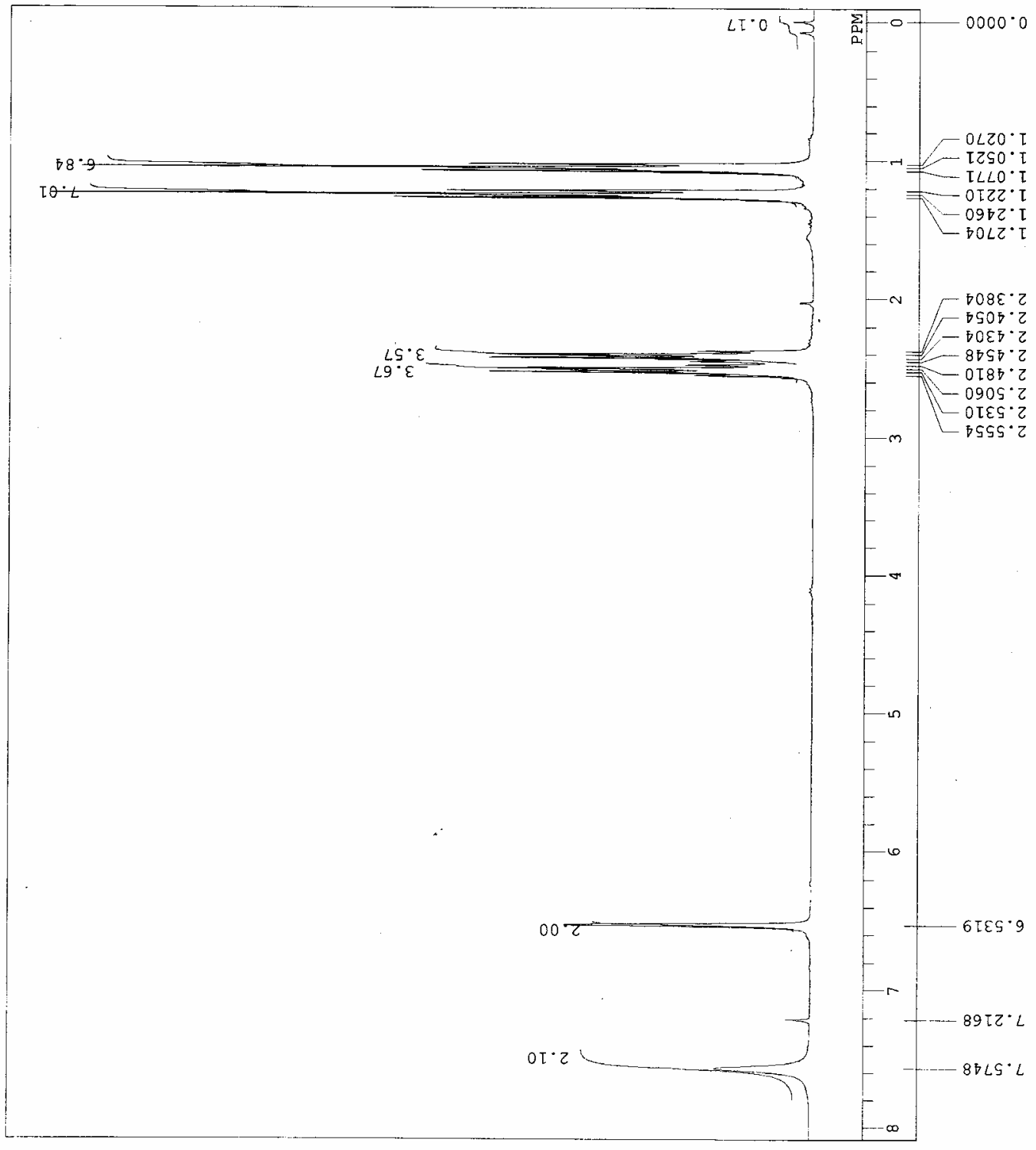




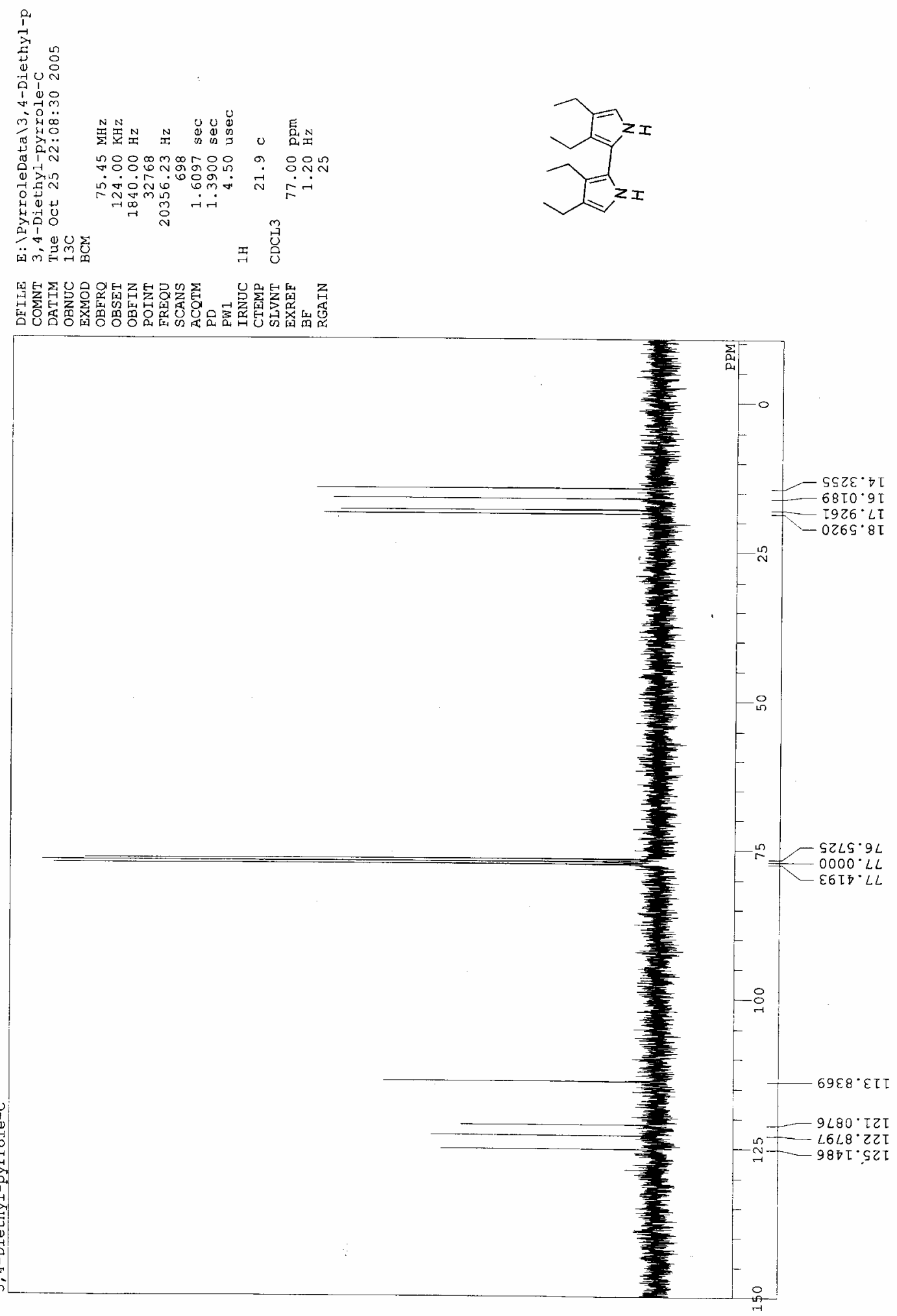




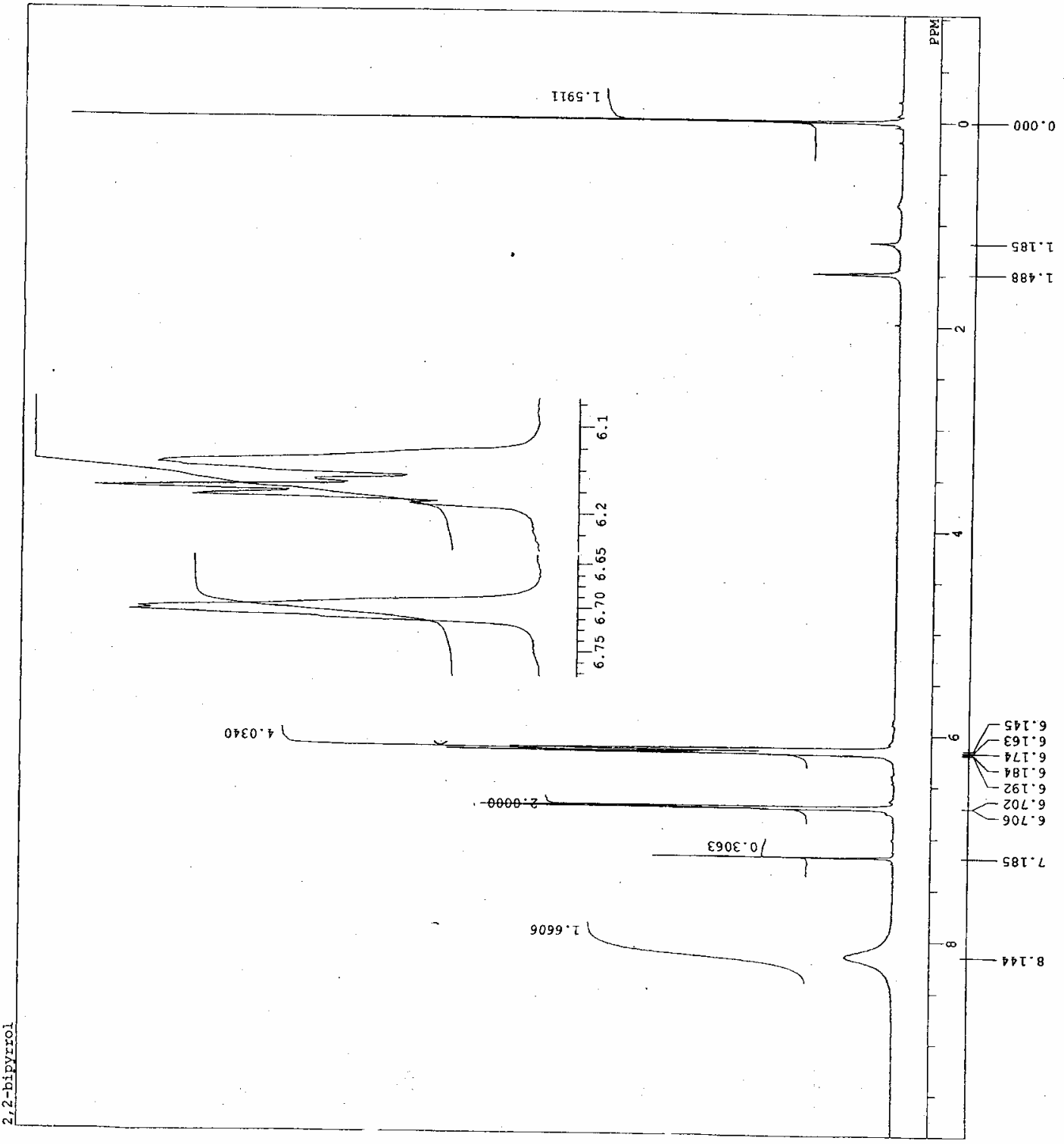




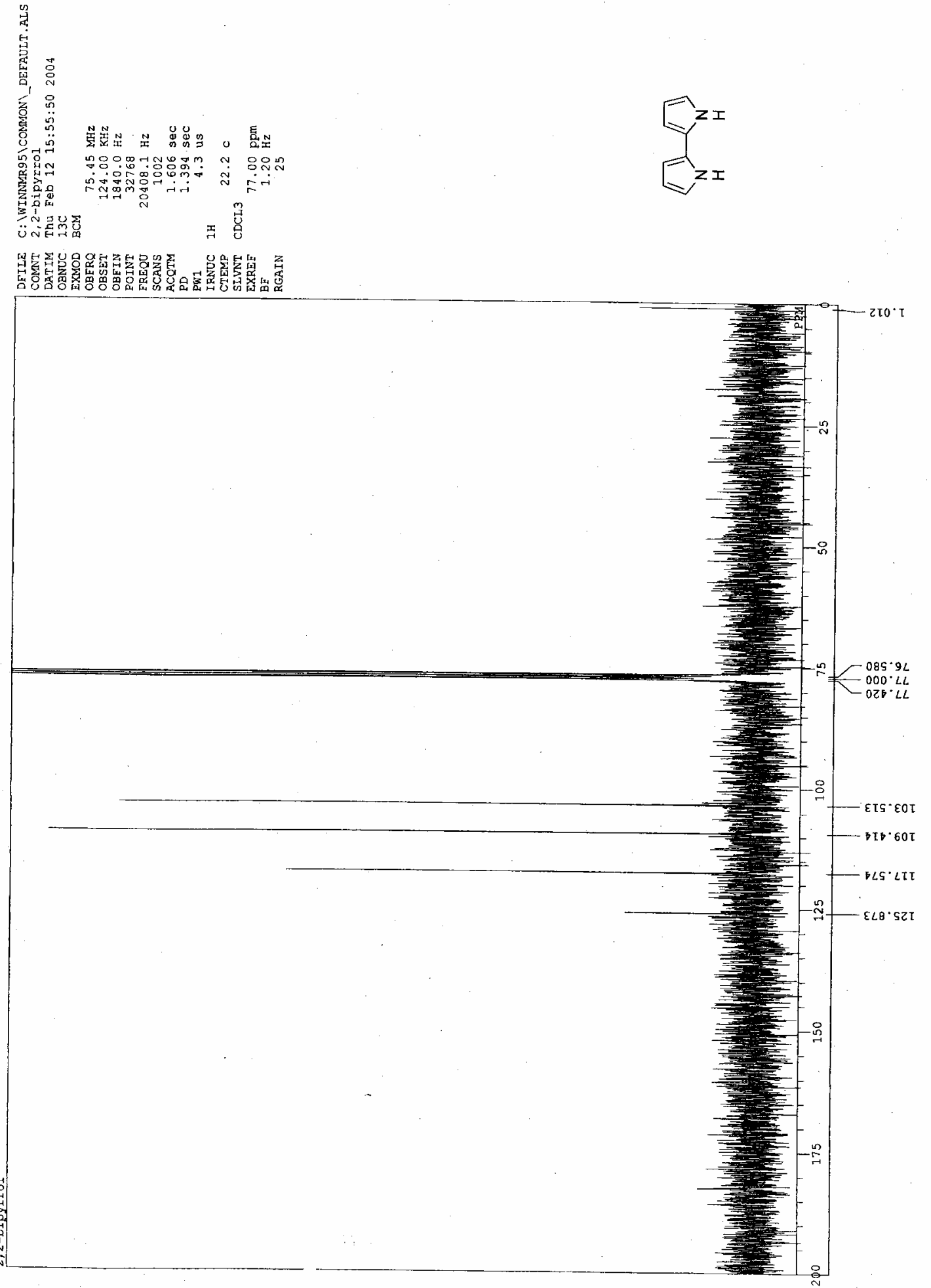




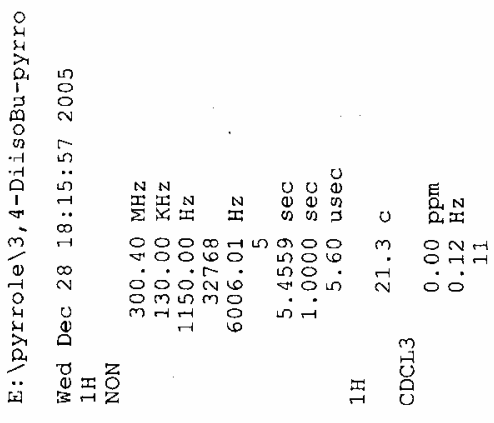

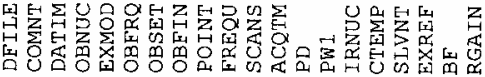
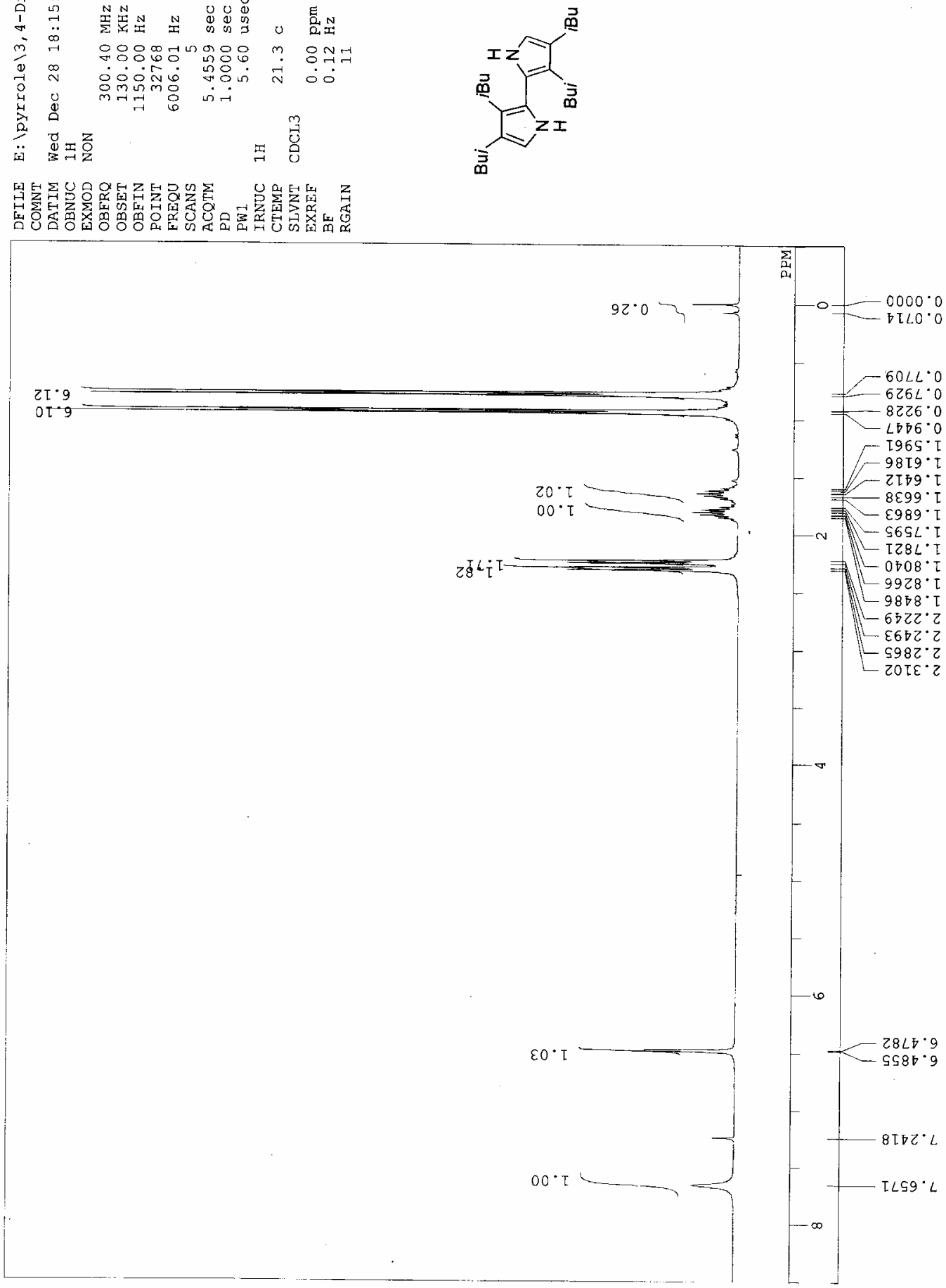

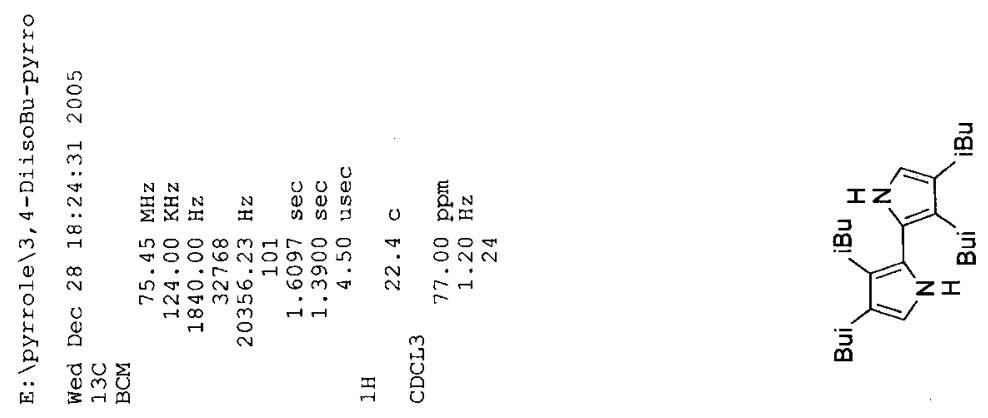

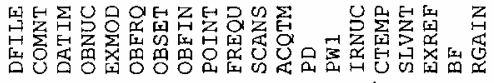

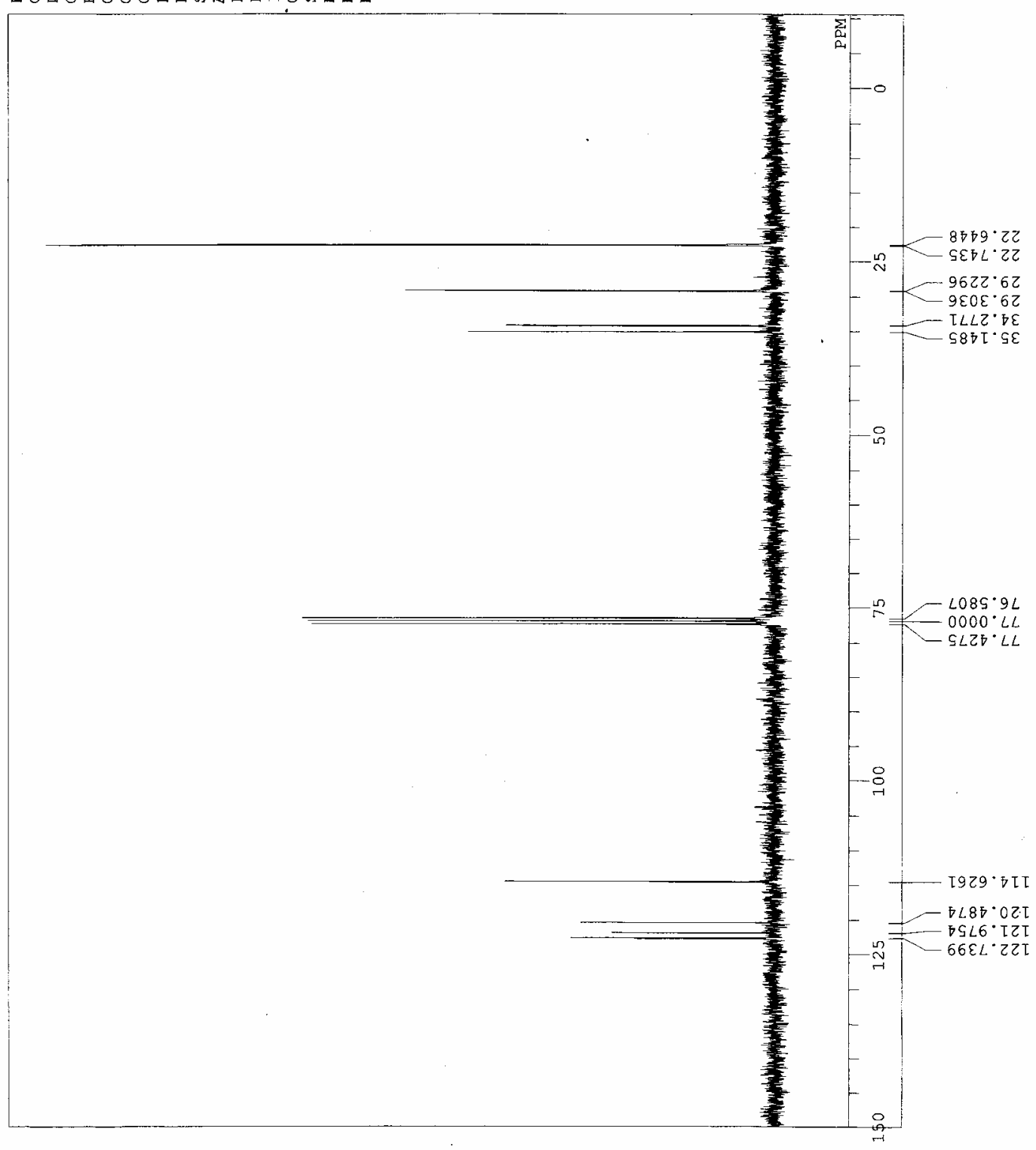



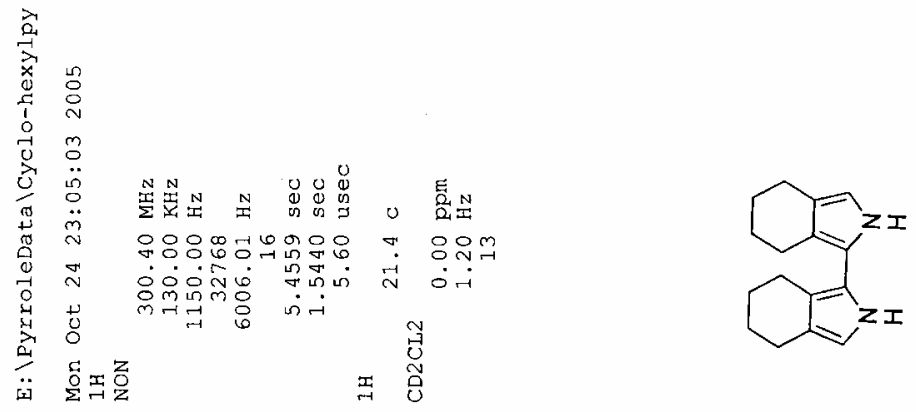

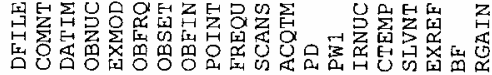

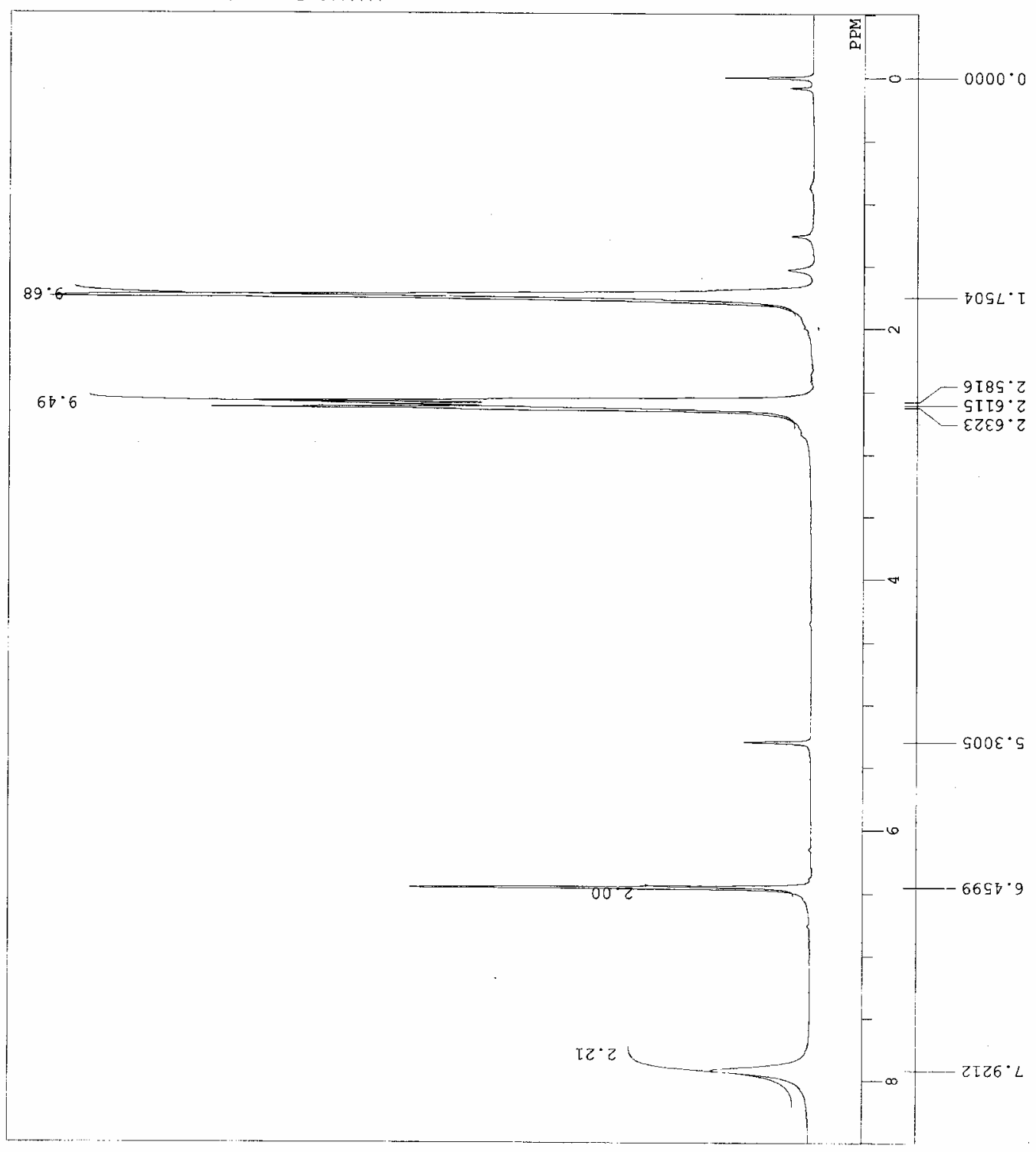




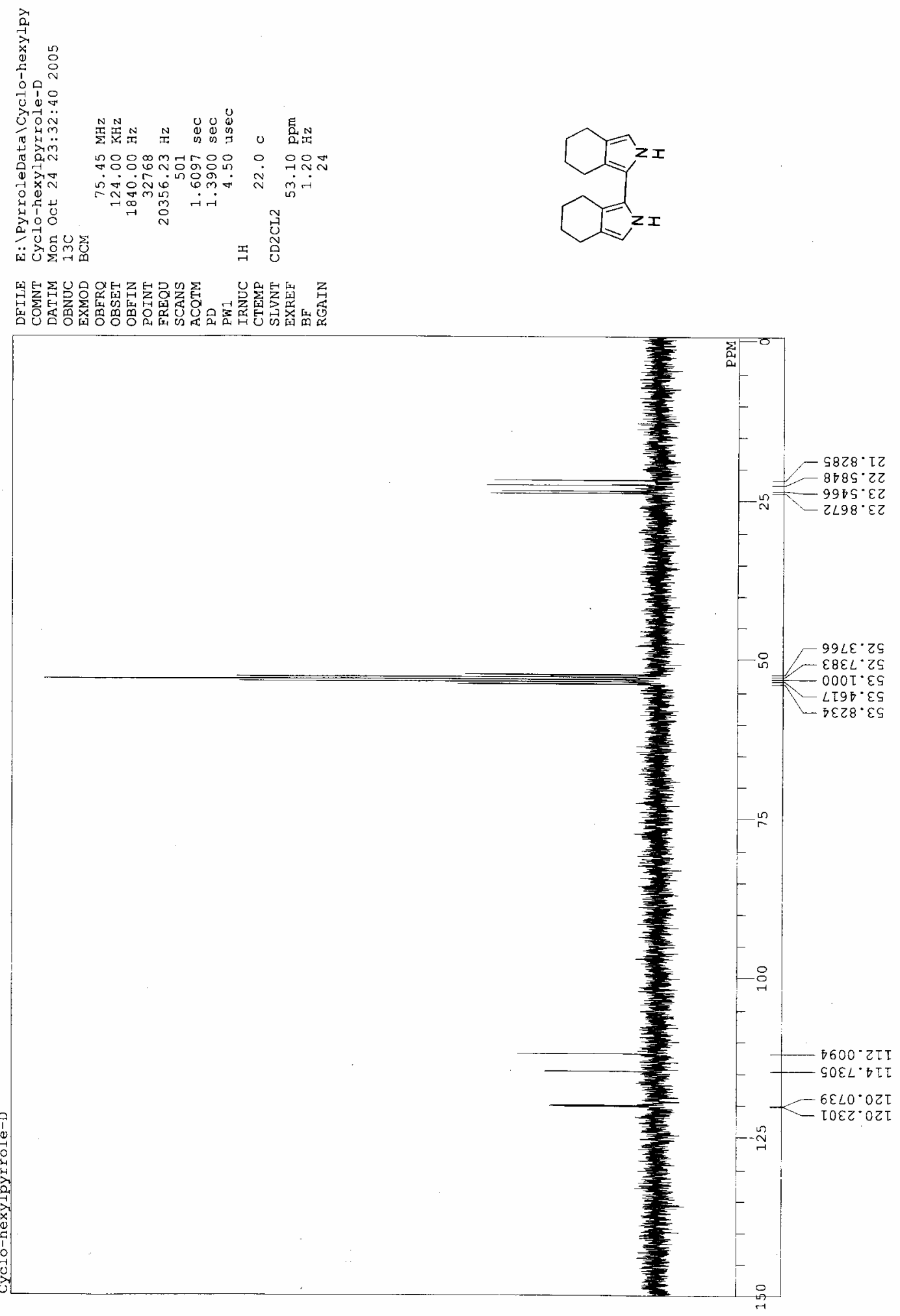



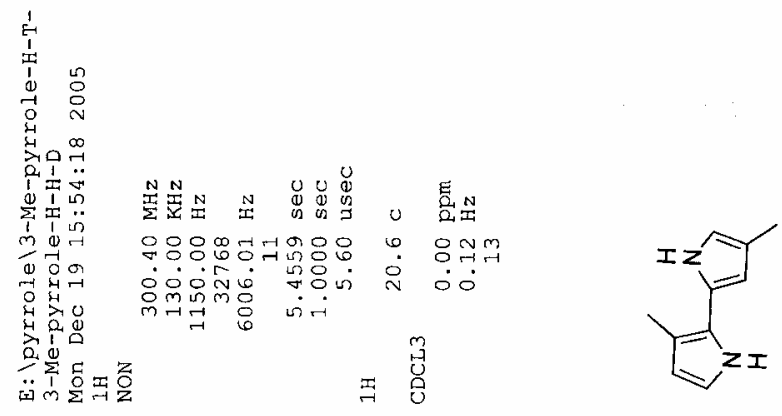

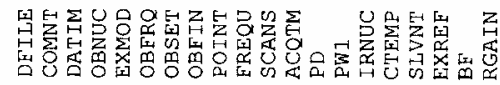

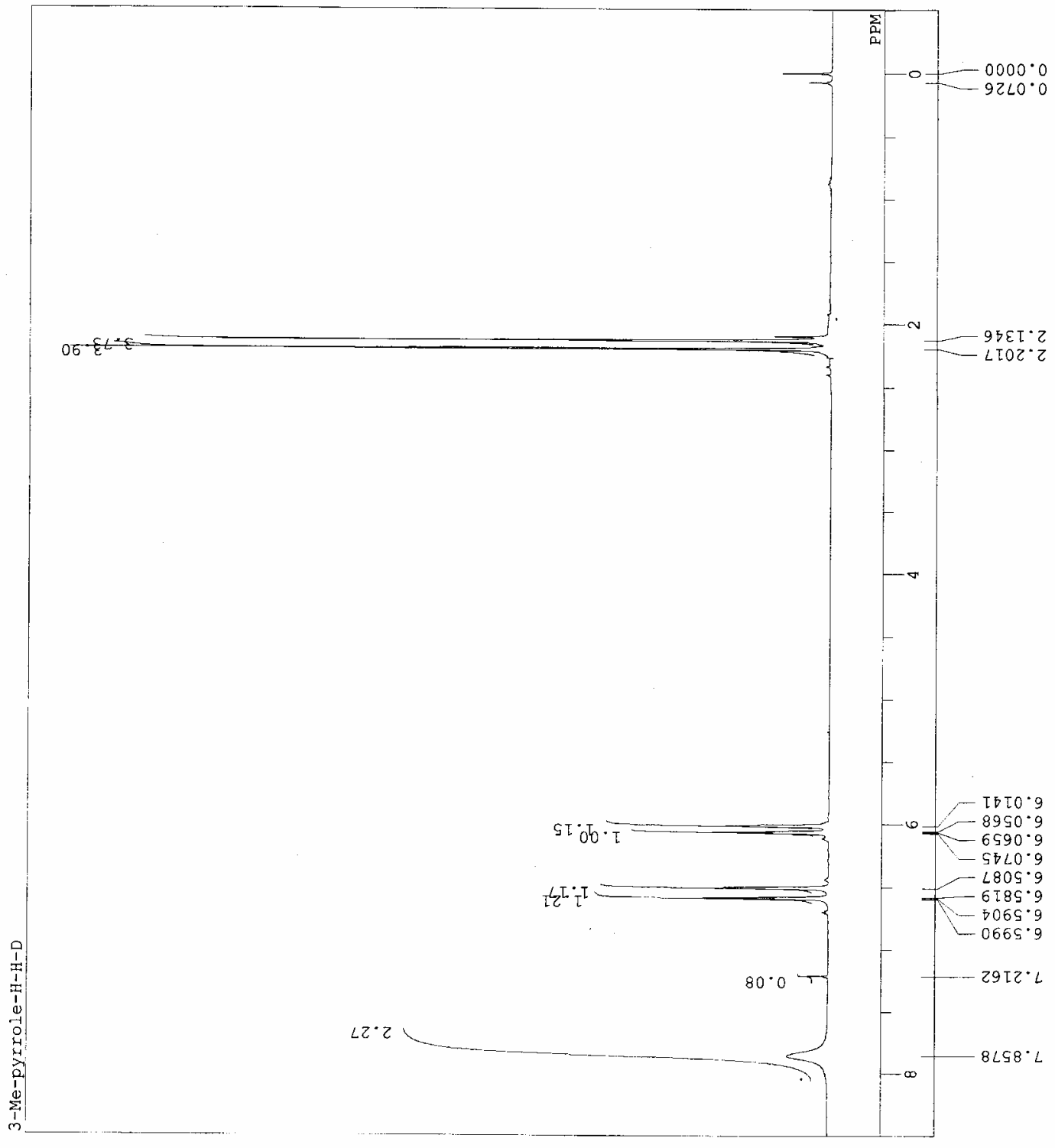



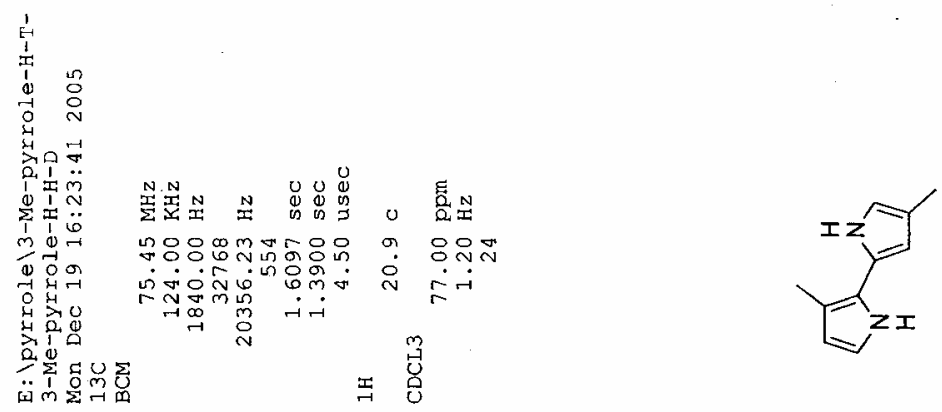

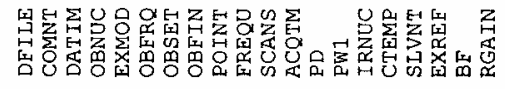

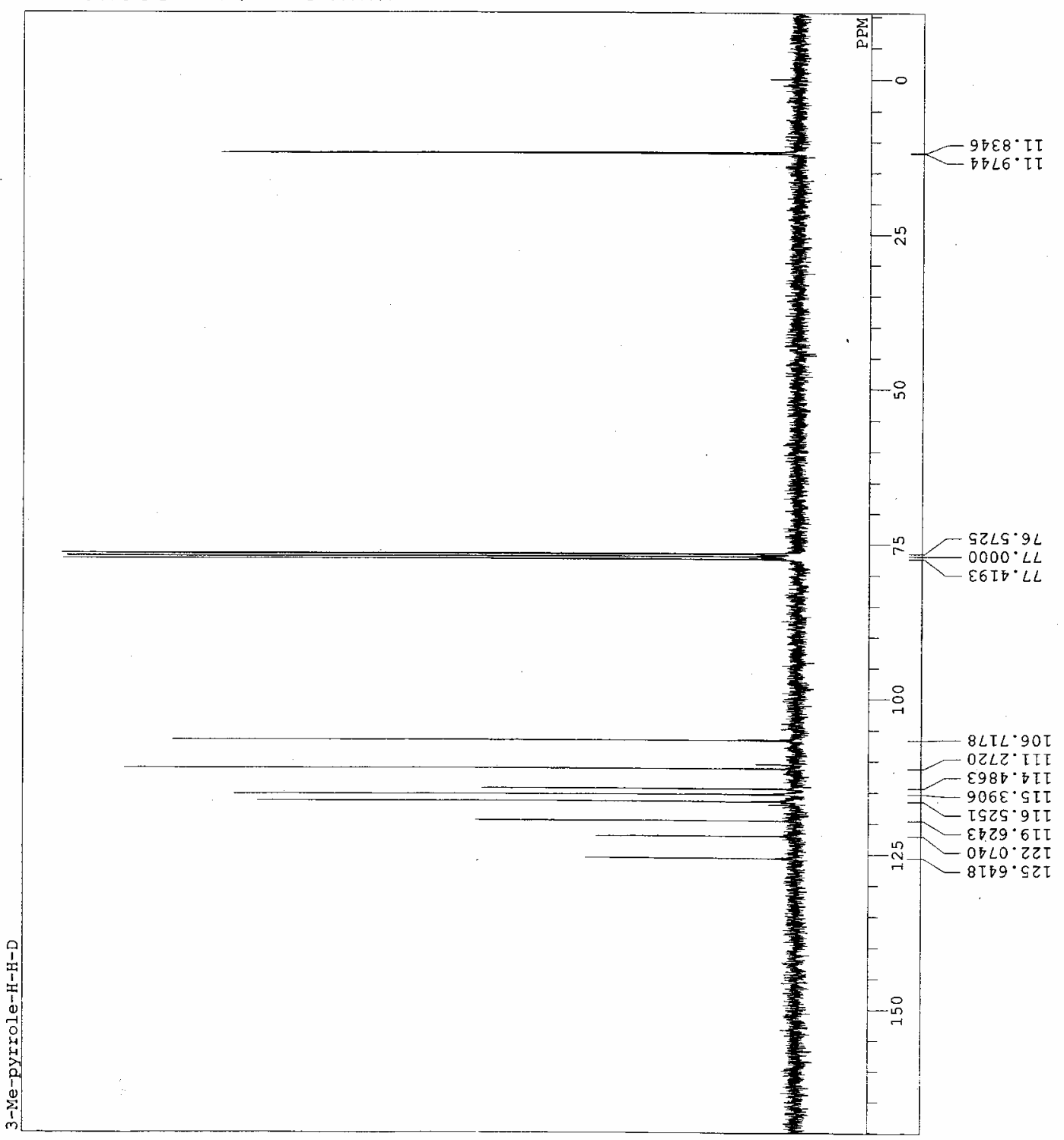




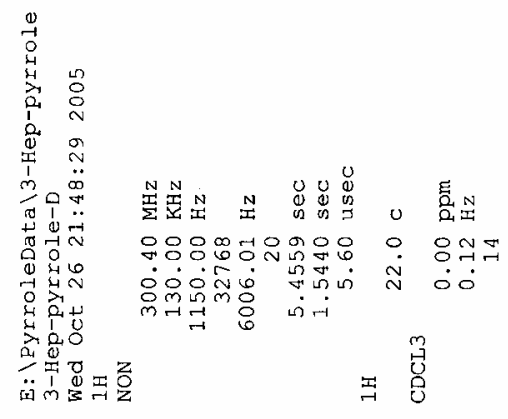

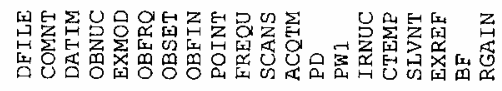
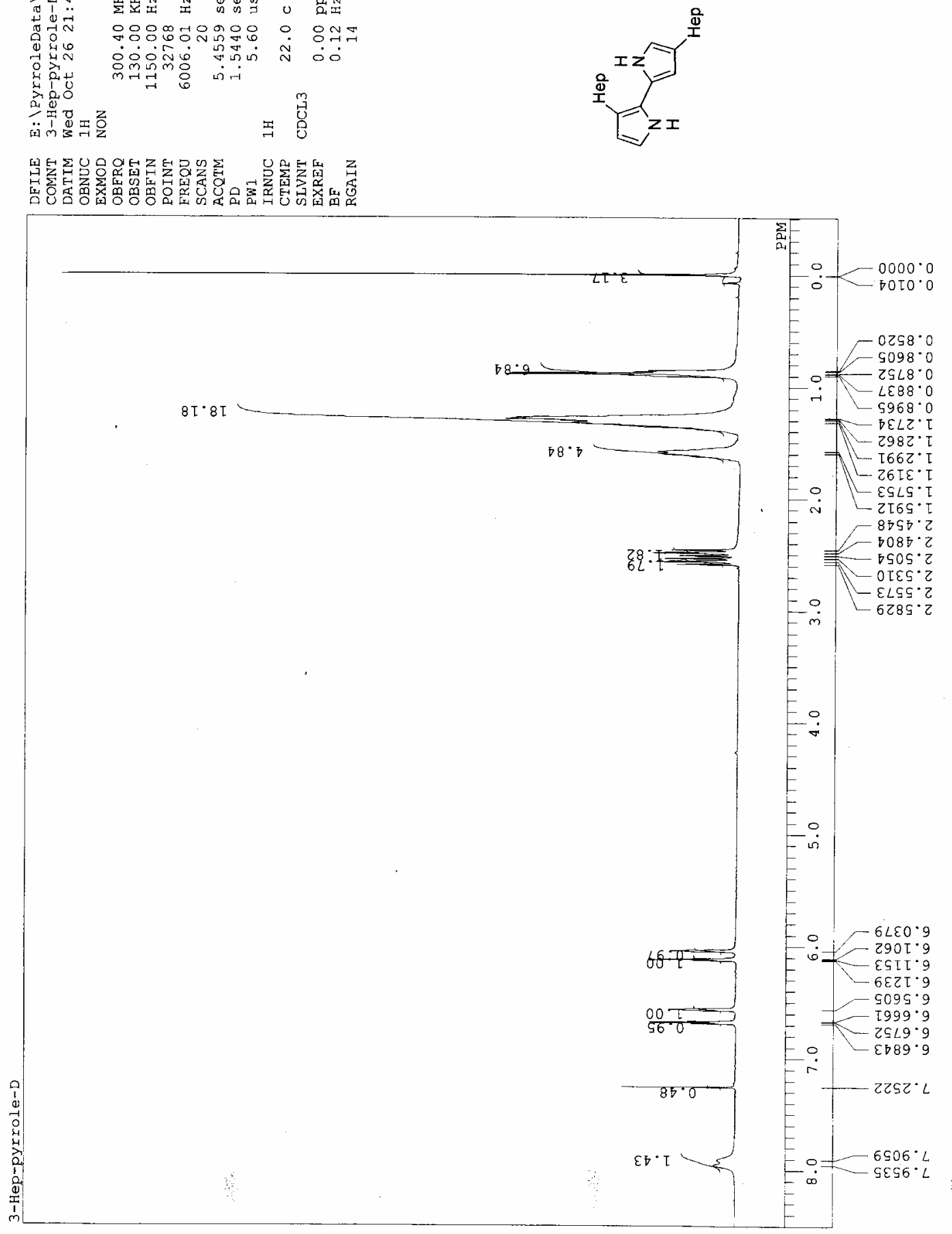

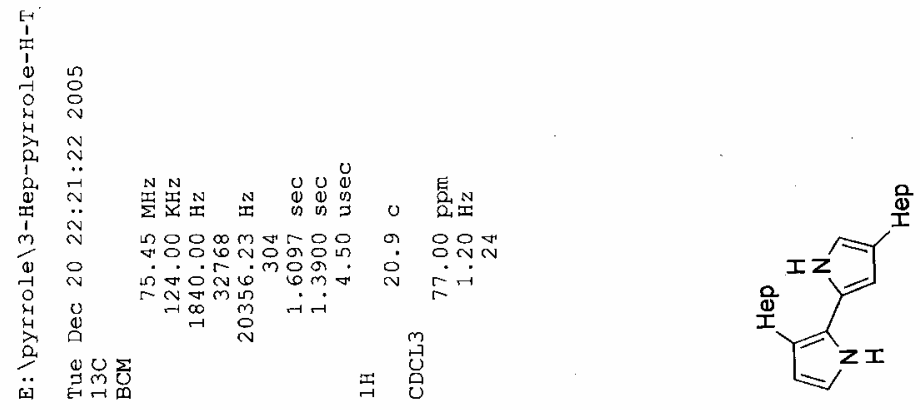

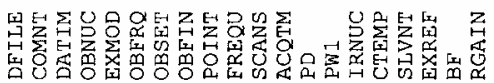

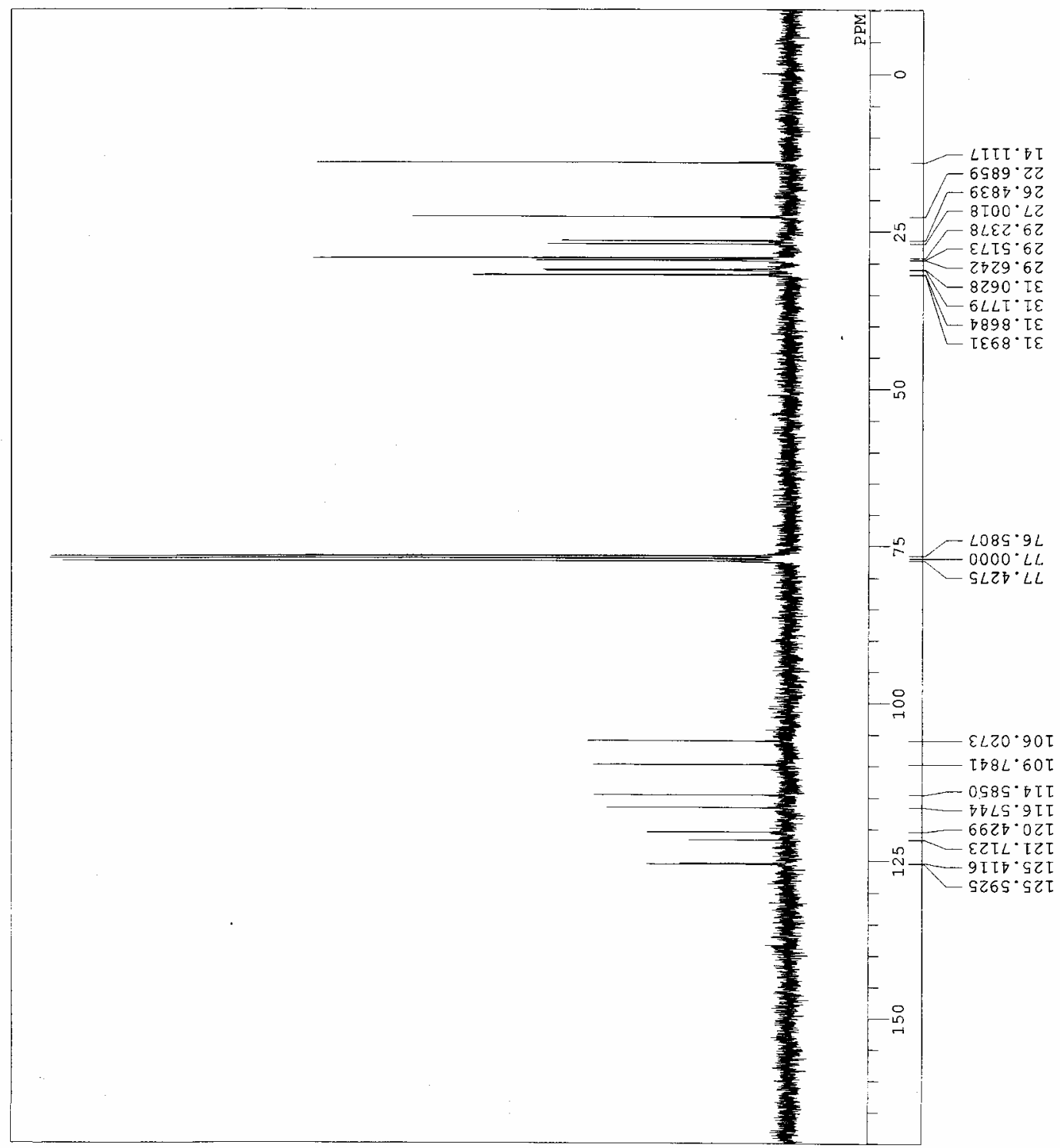




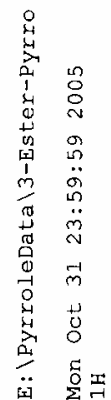

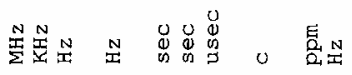

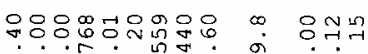

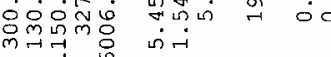

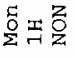

孚

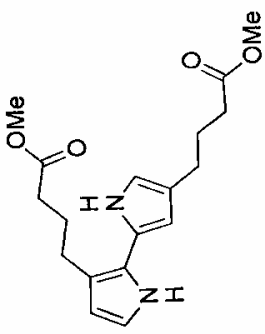

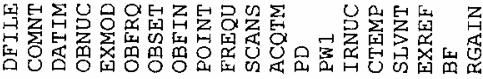

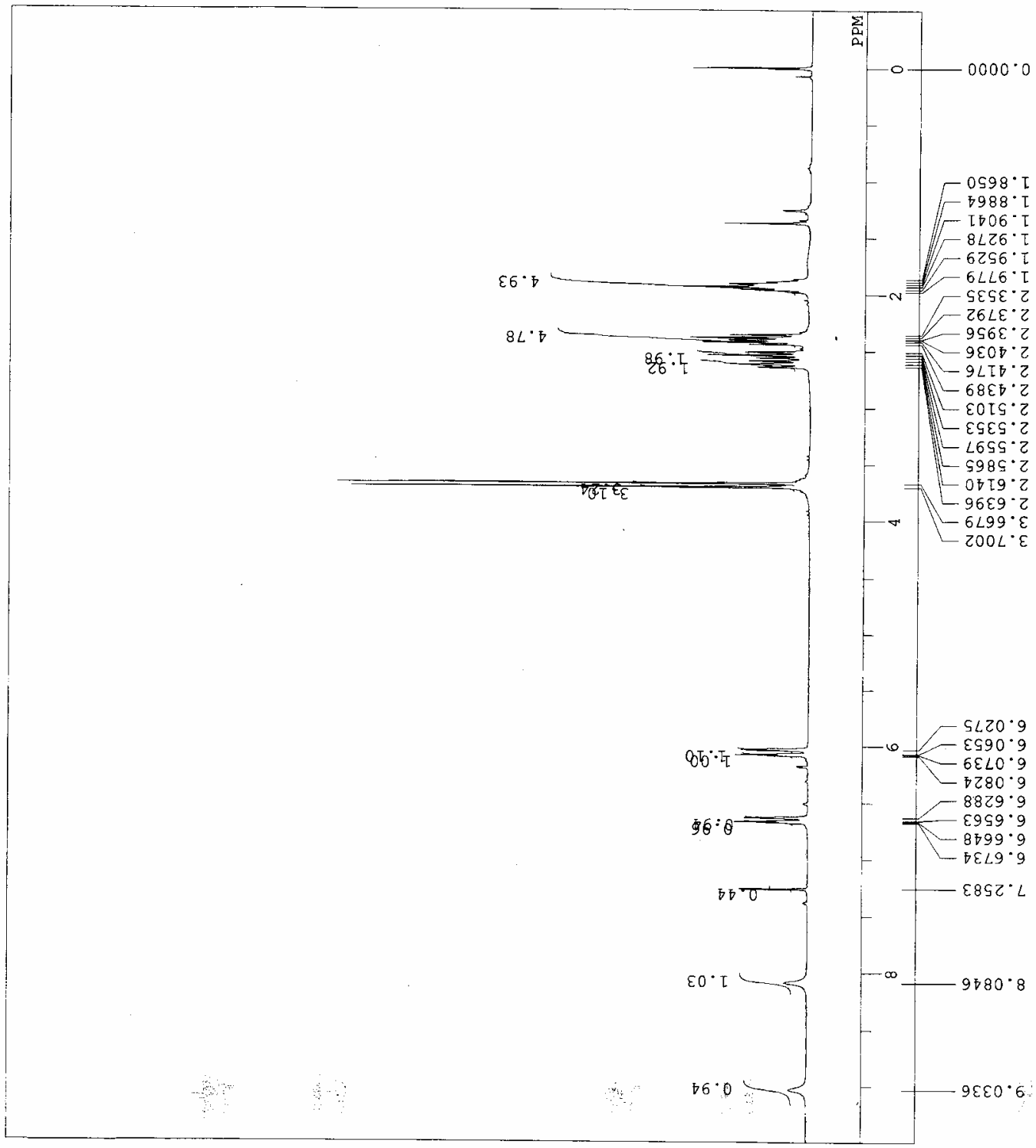



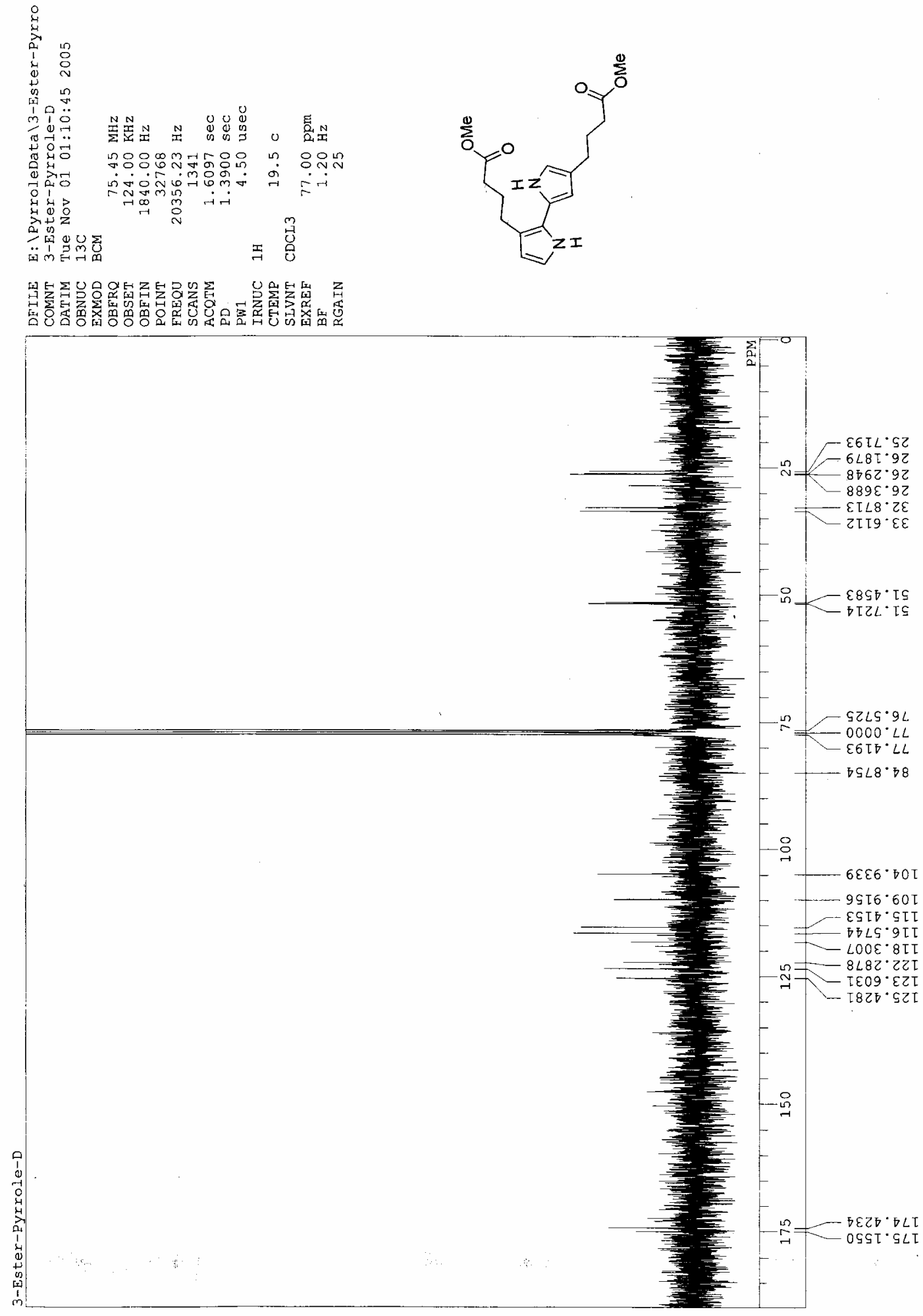


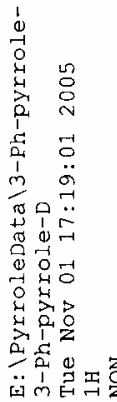

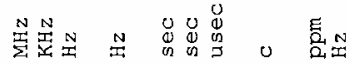

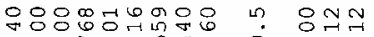

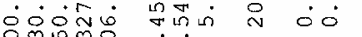

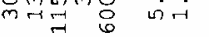

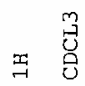

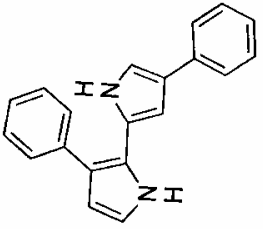

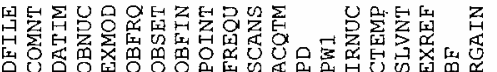

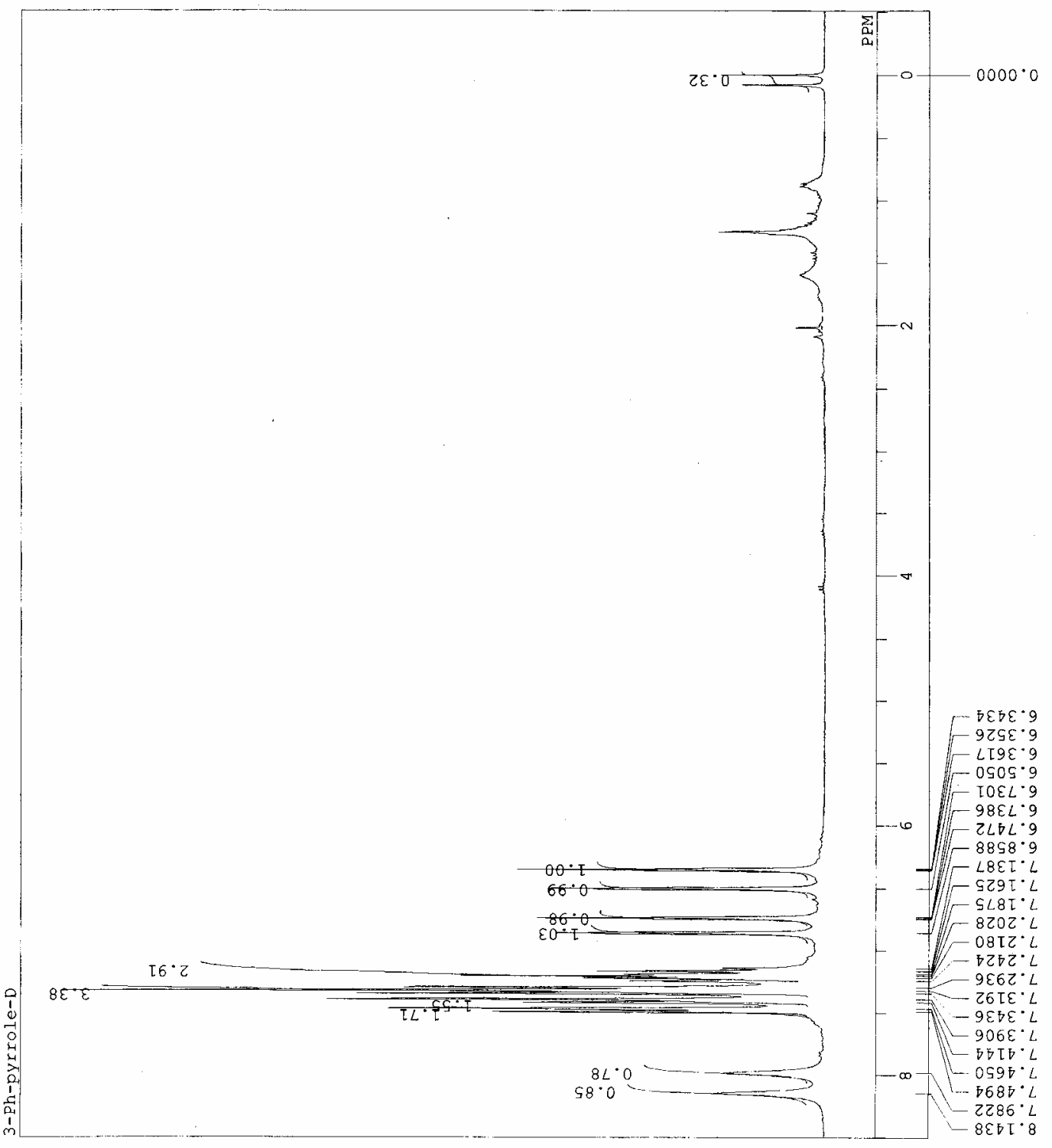



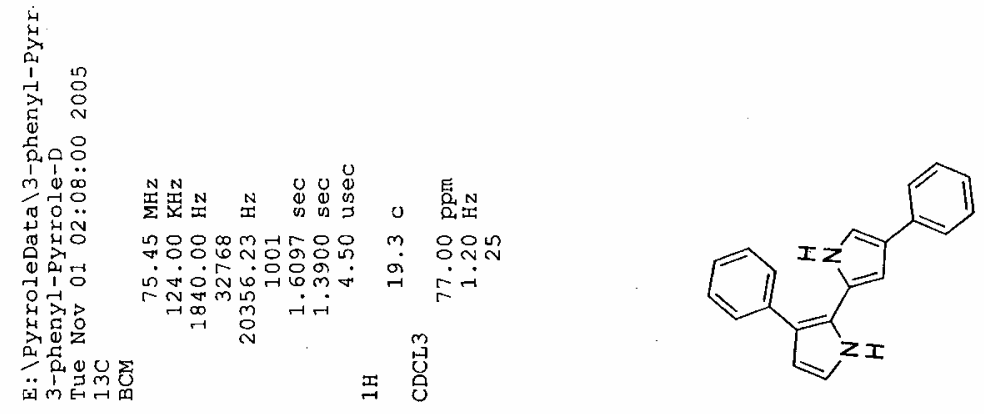

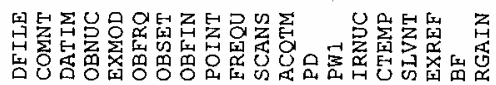

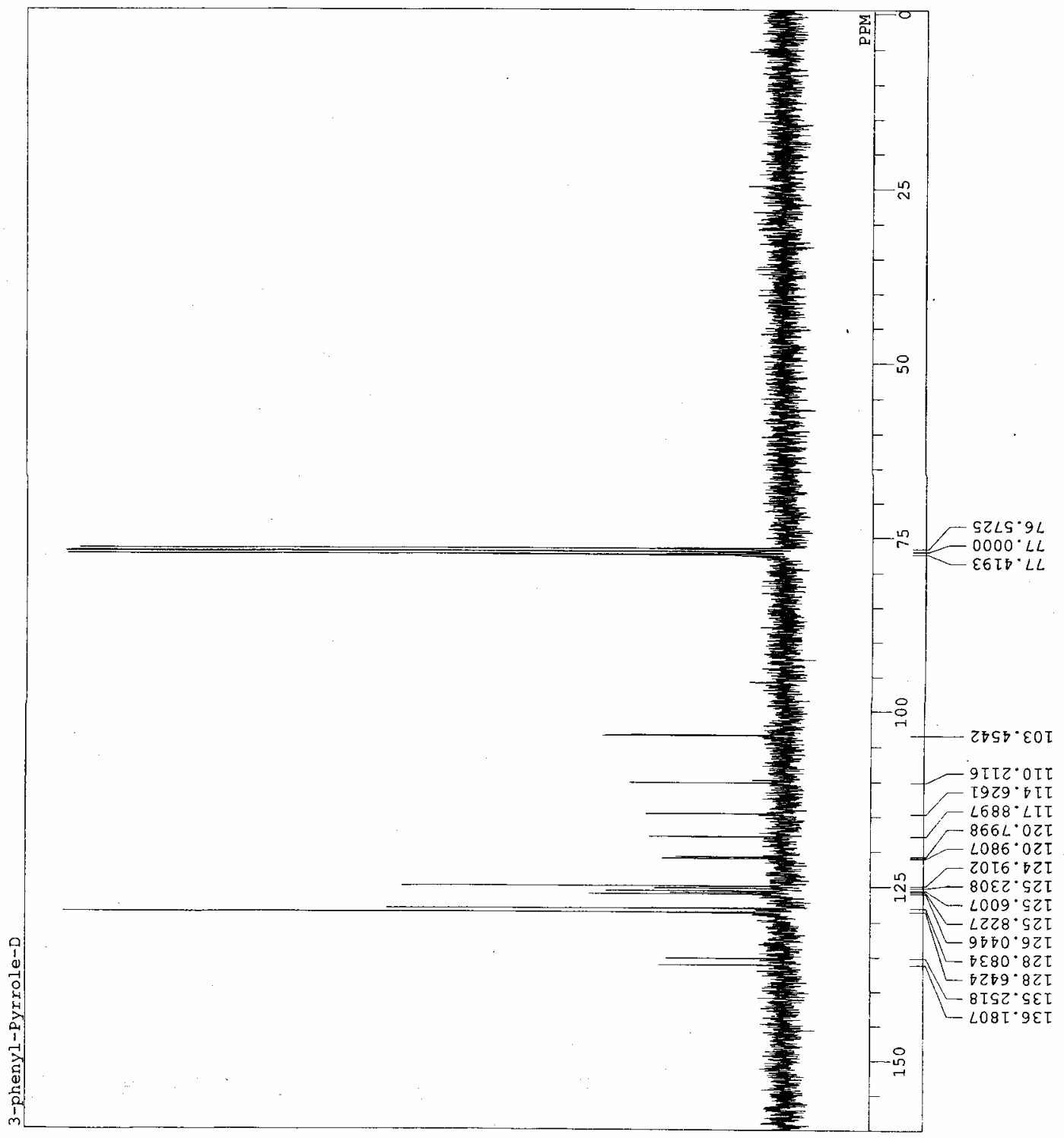



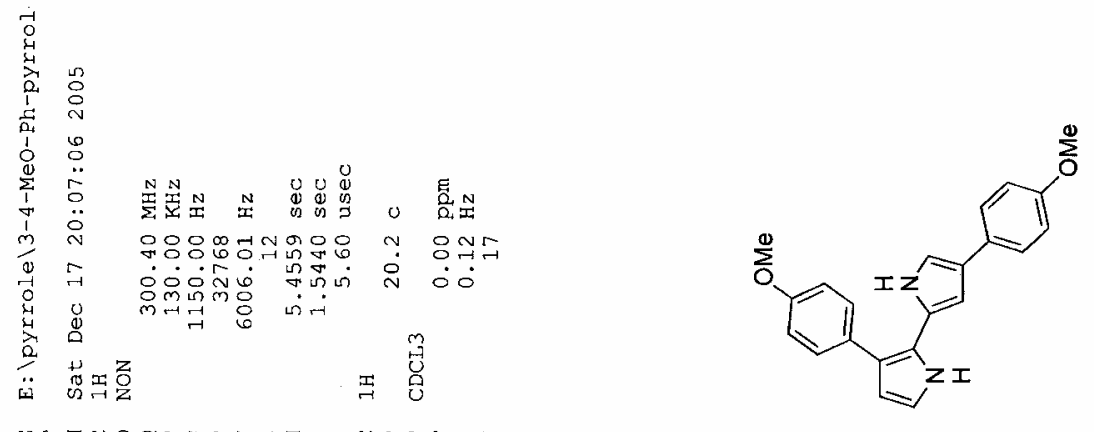

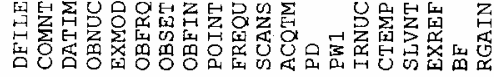

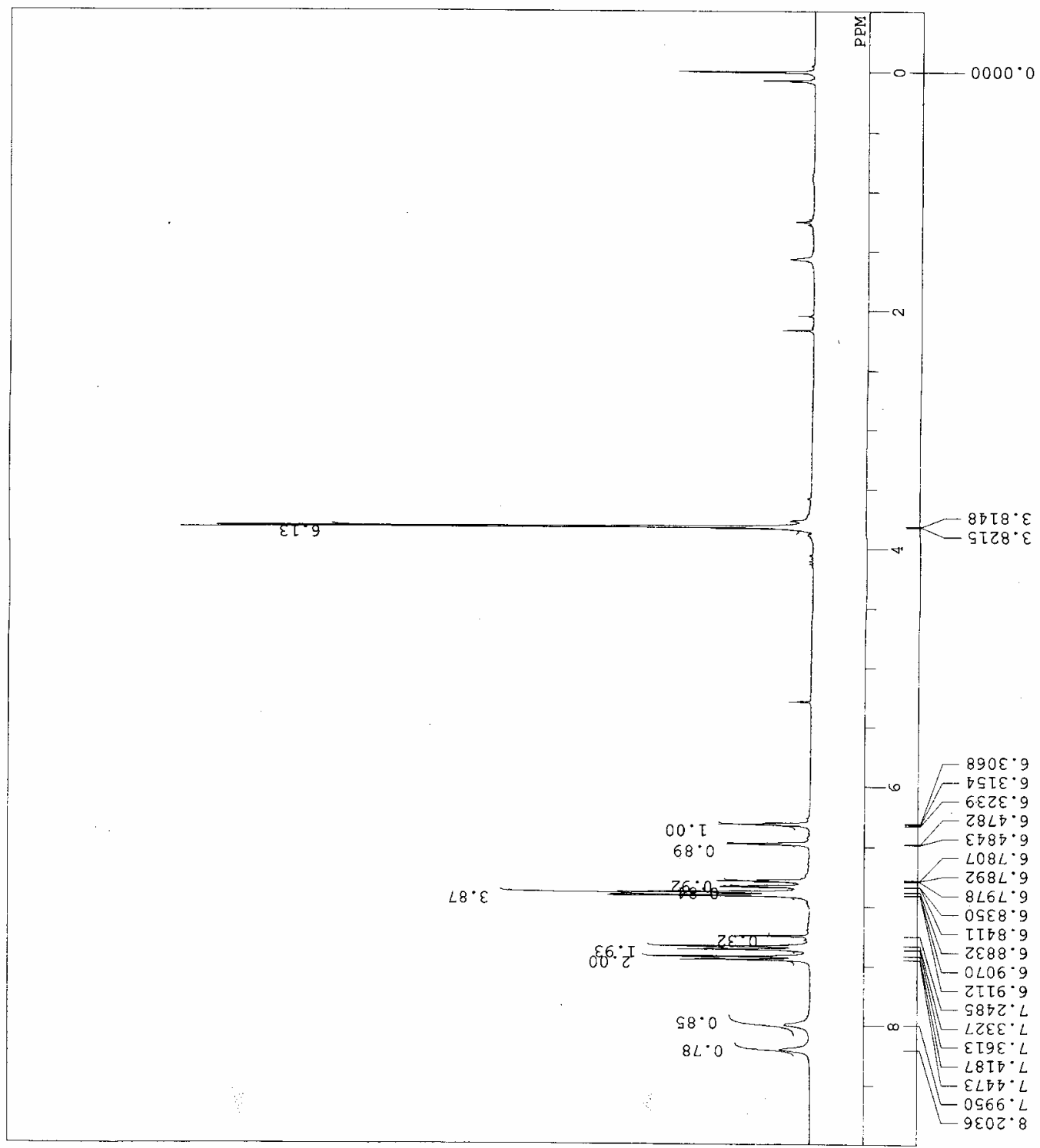



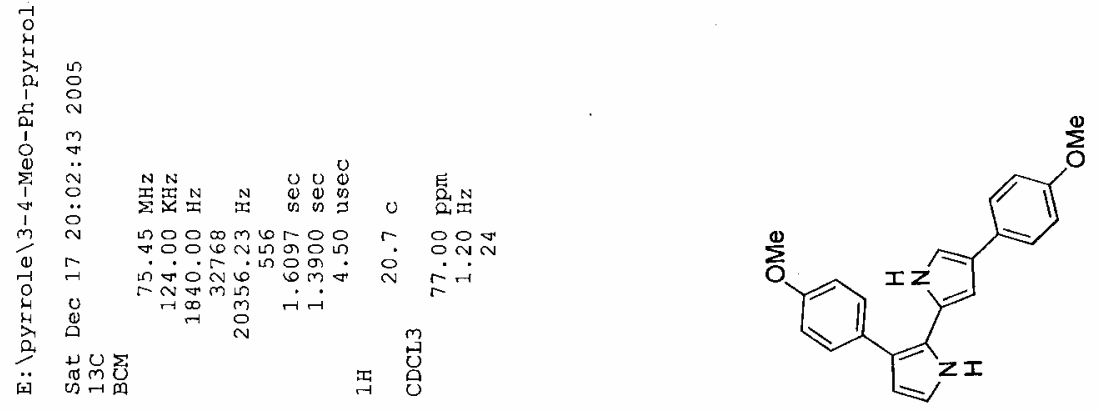

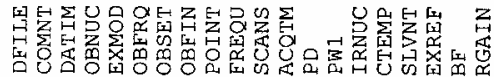

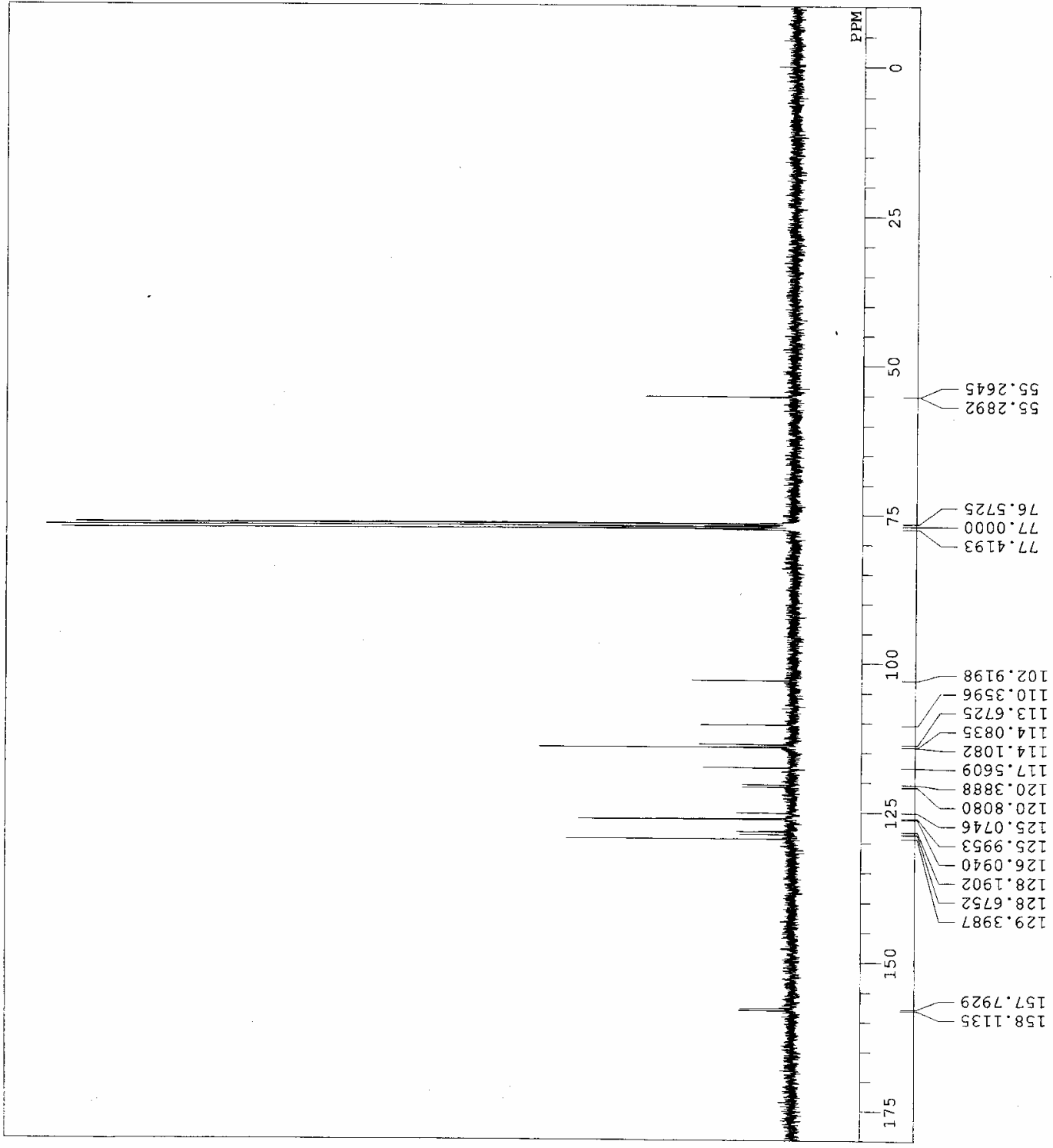




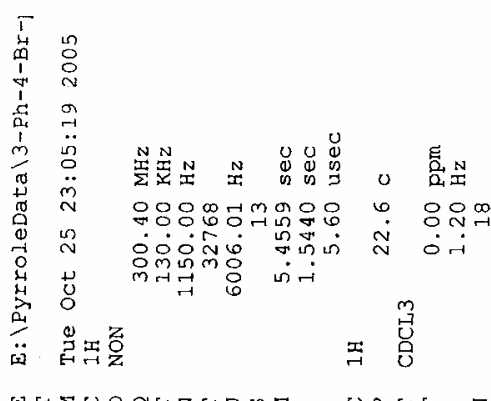

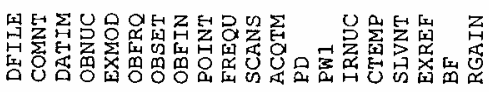
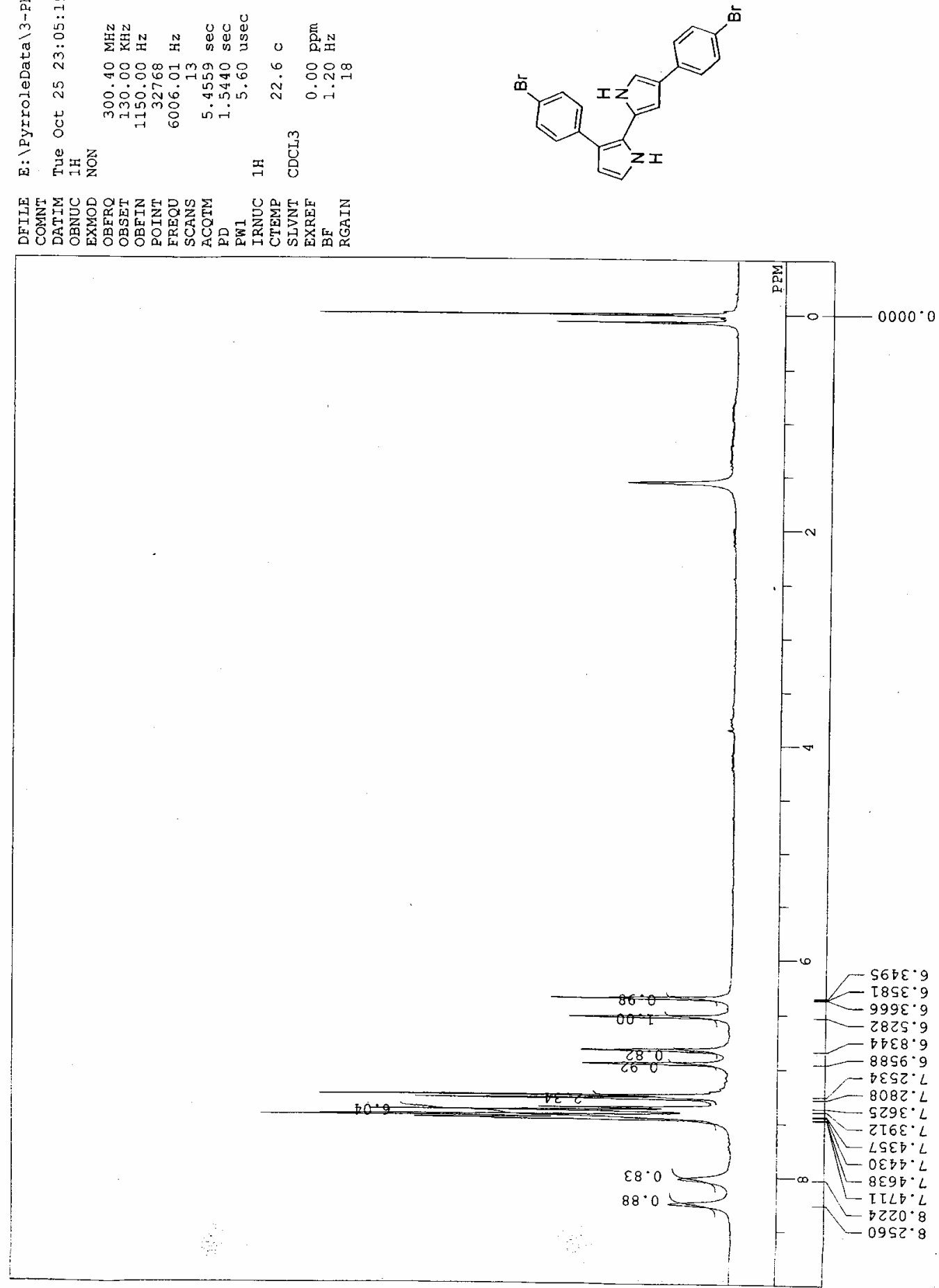


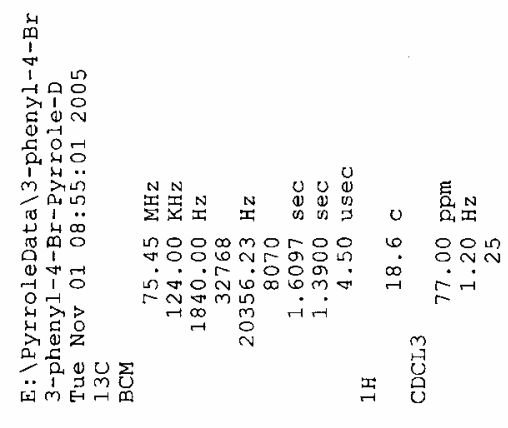

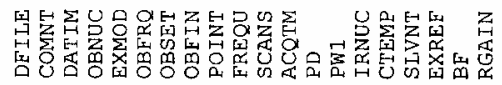
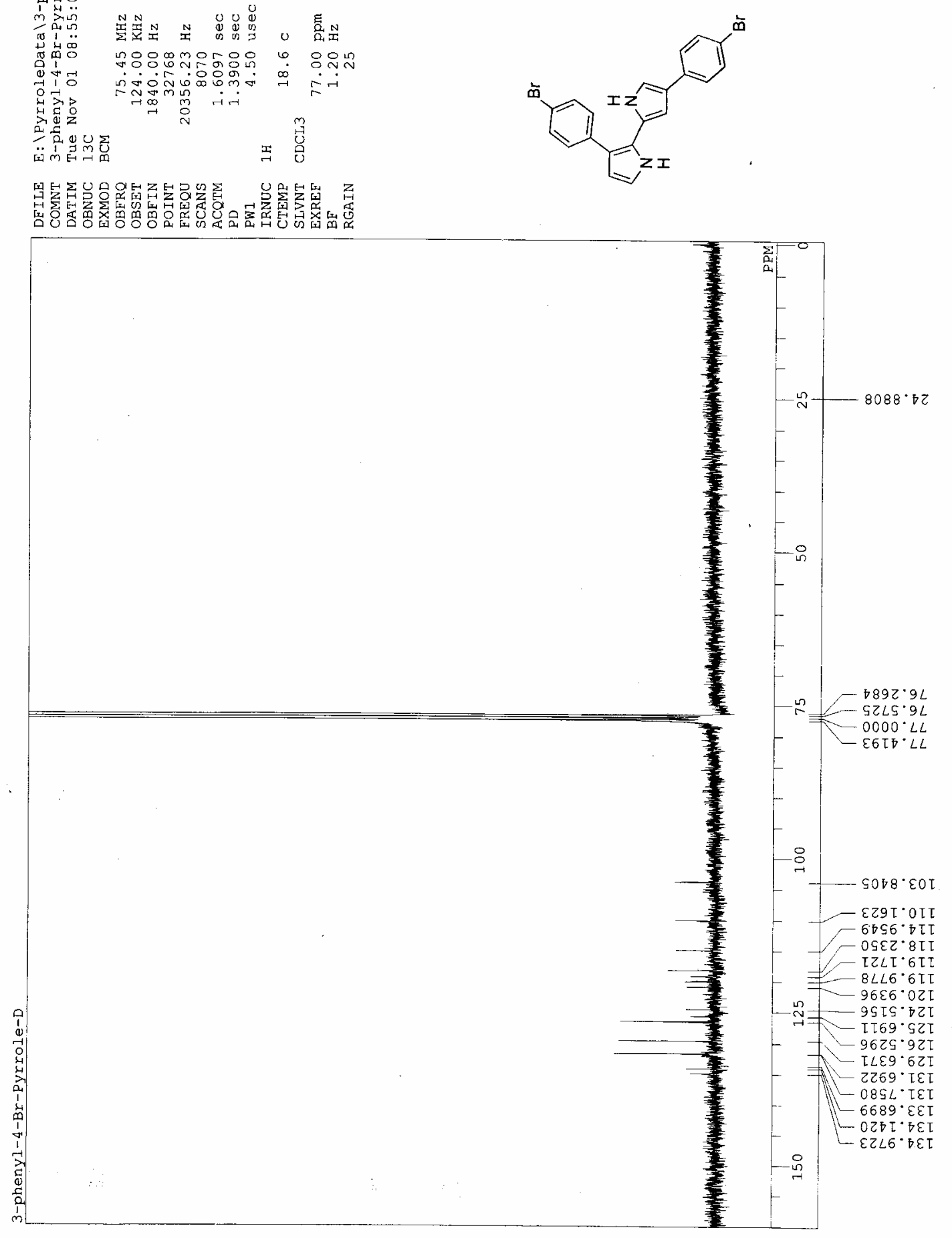


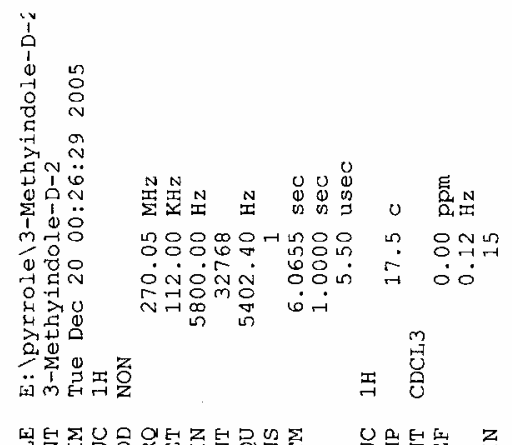

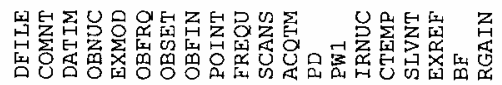
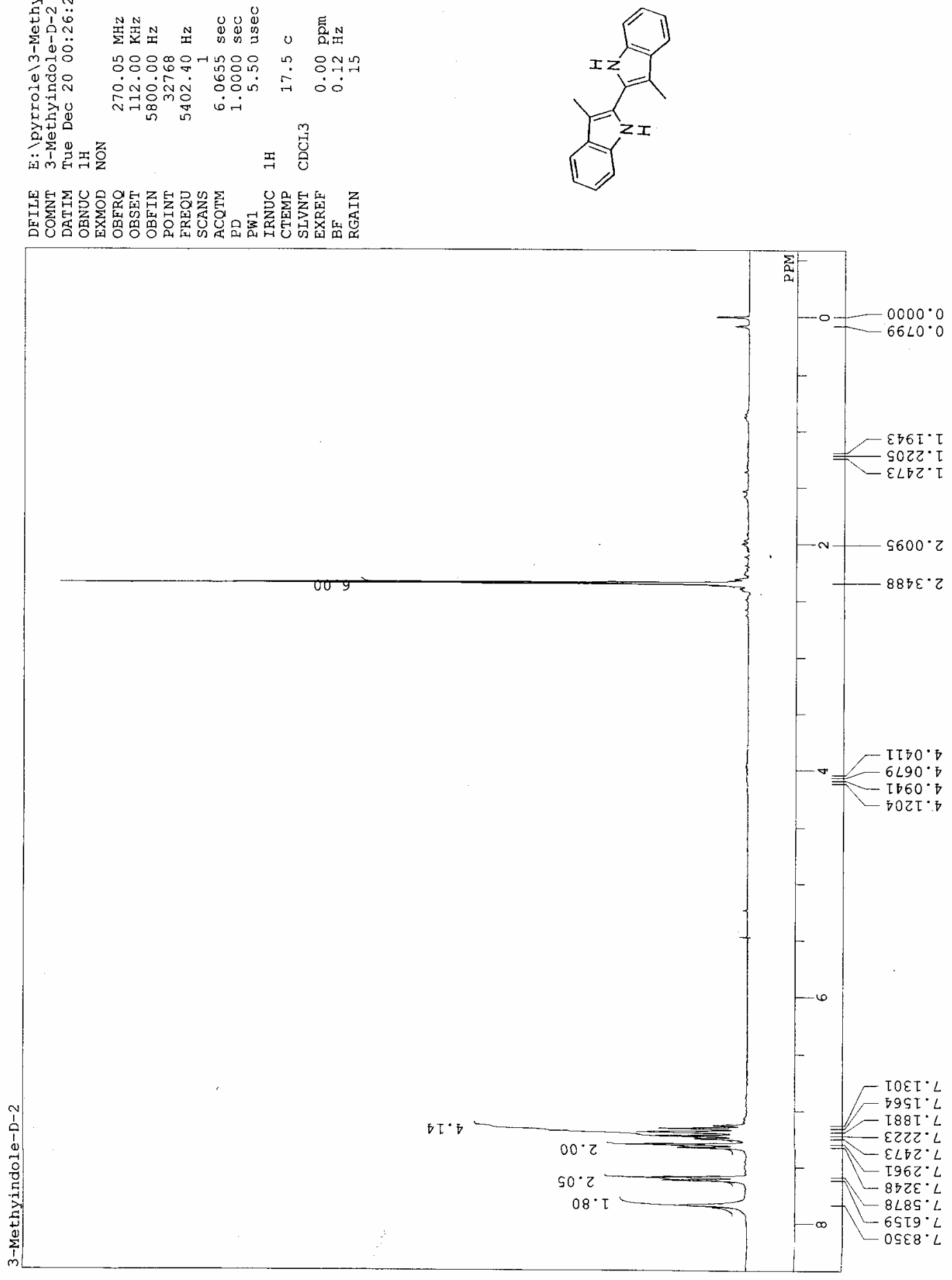


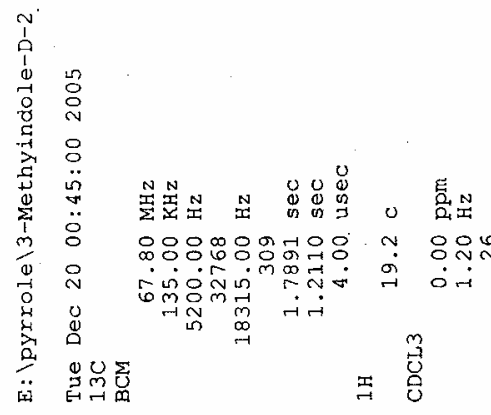

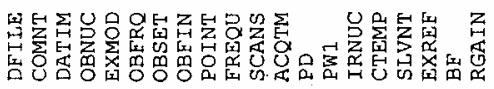
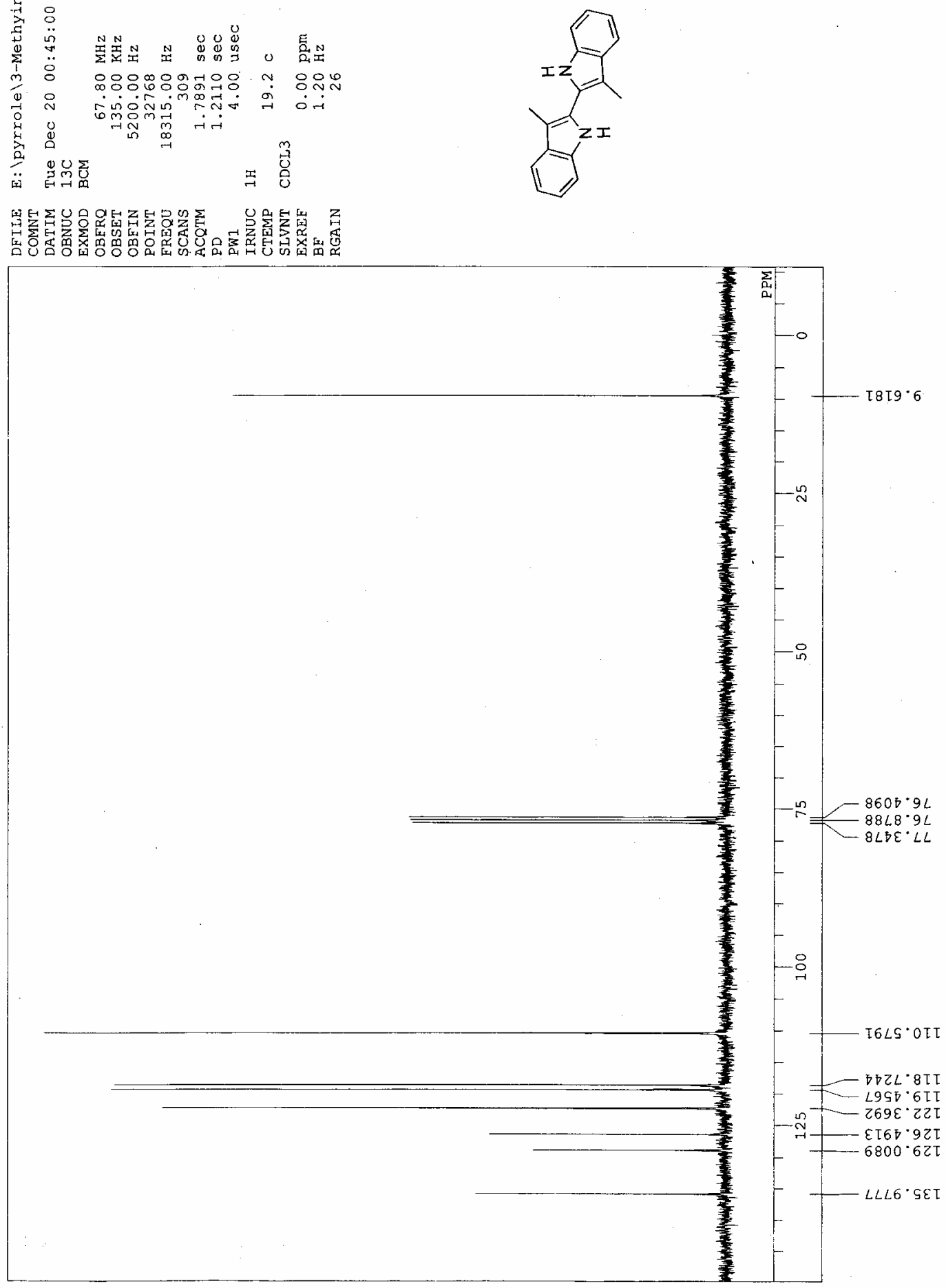

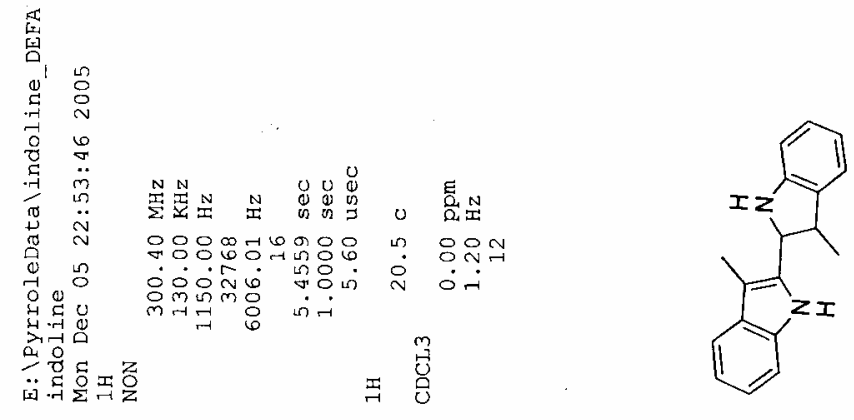

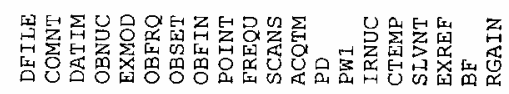

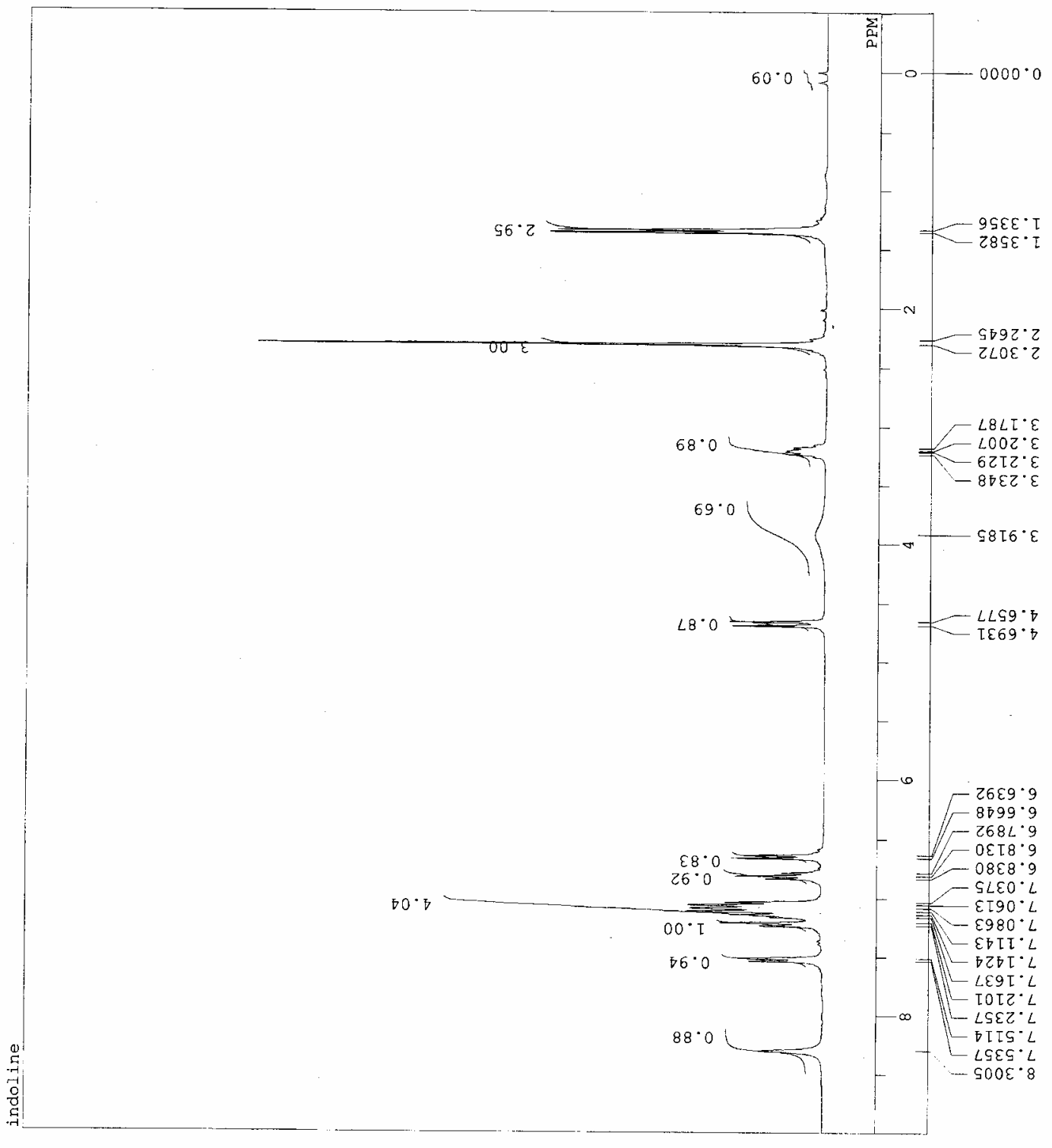



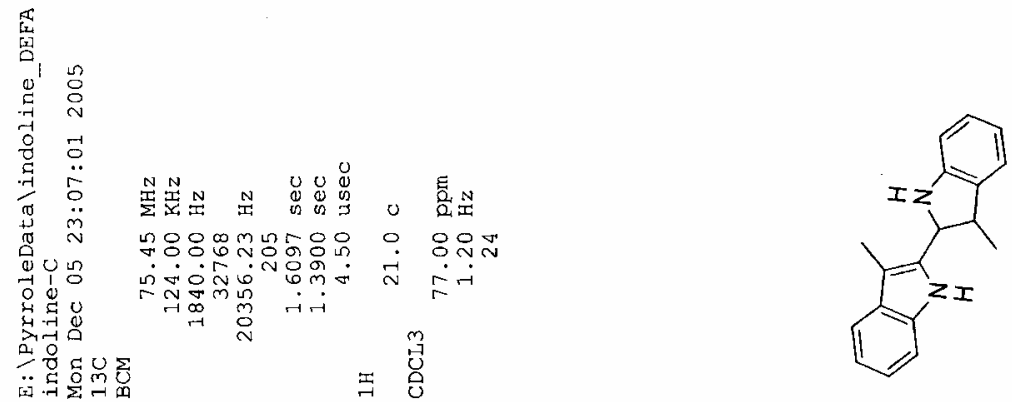

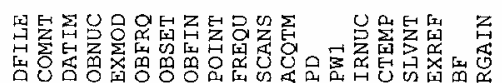

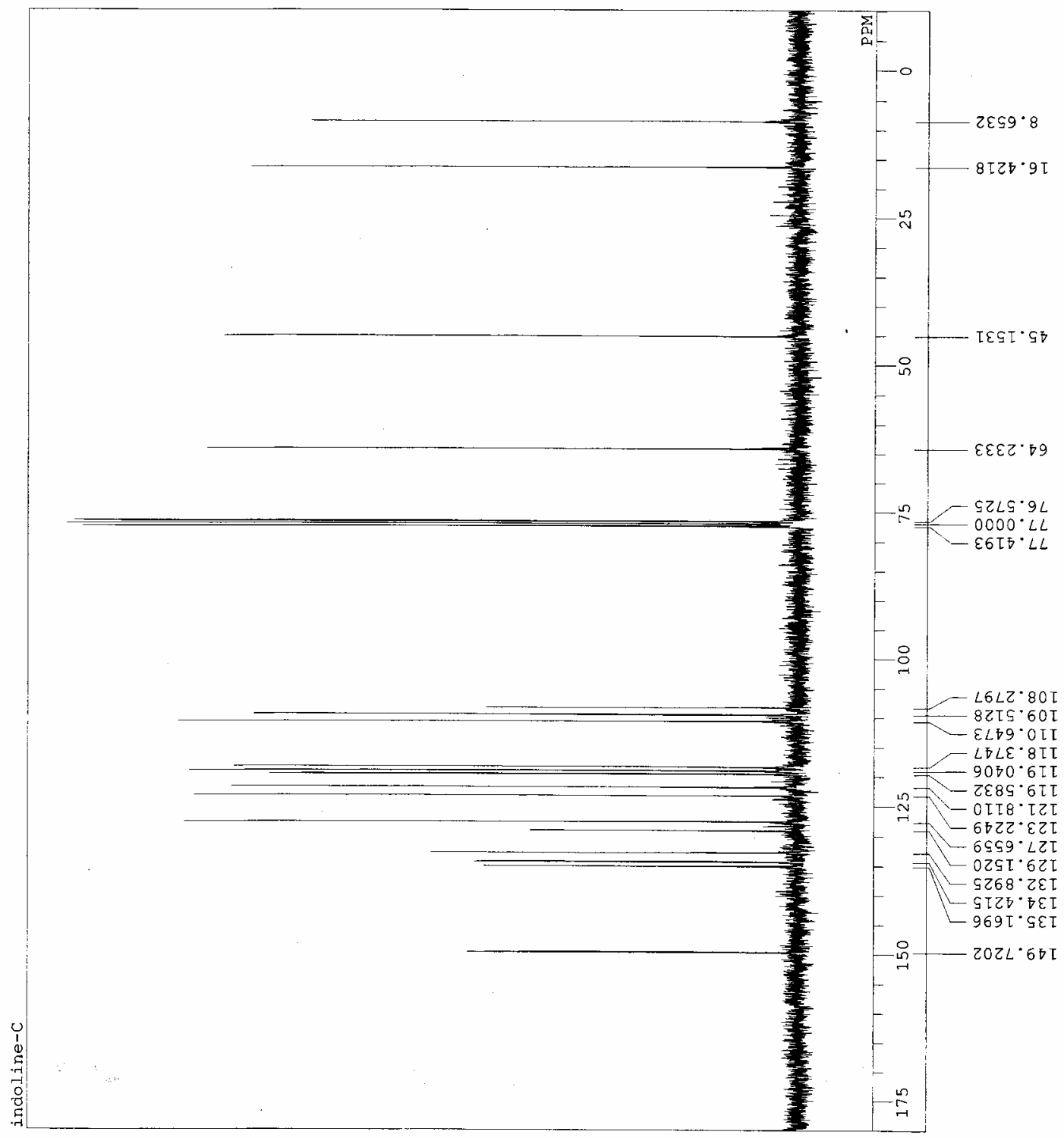



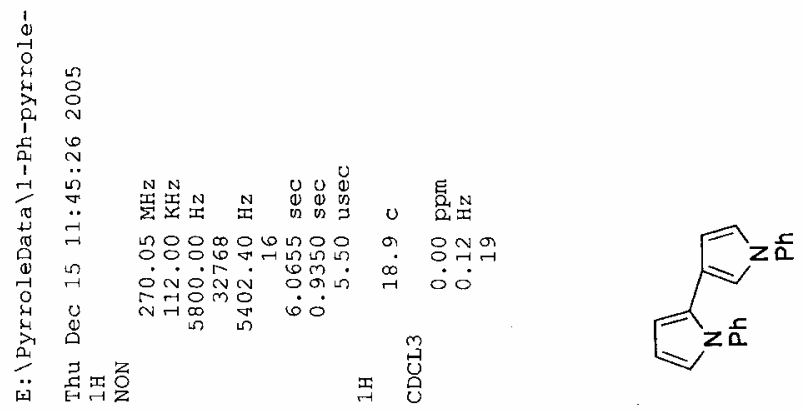

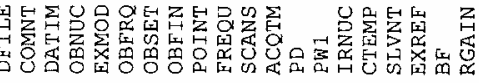

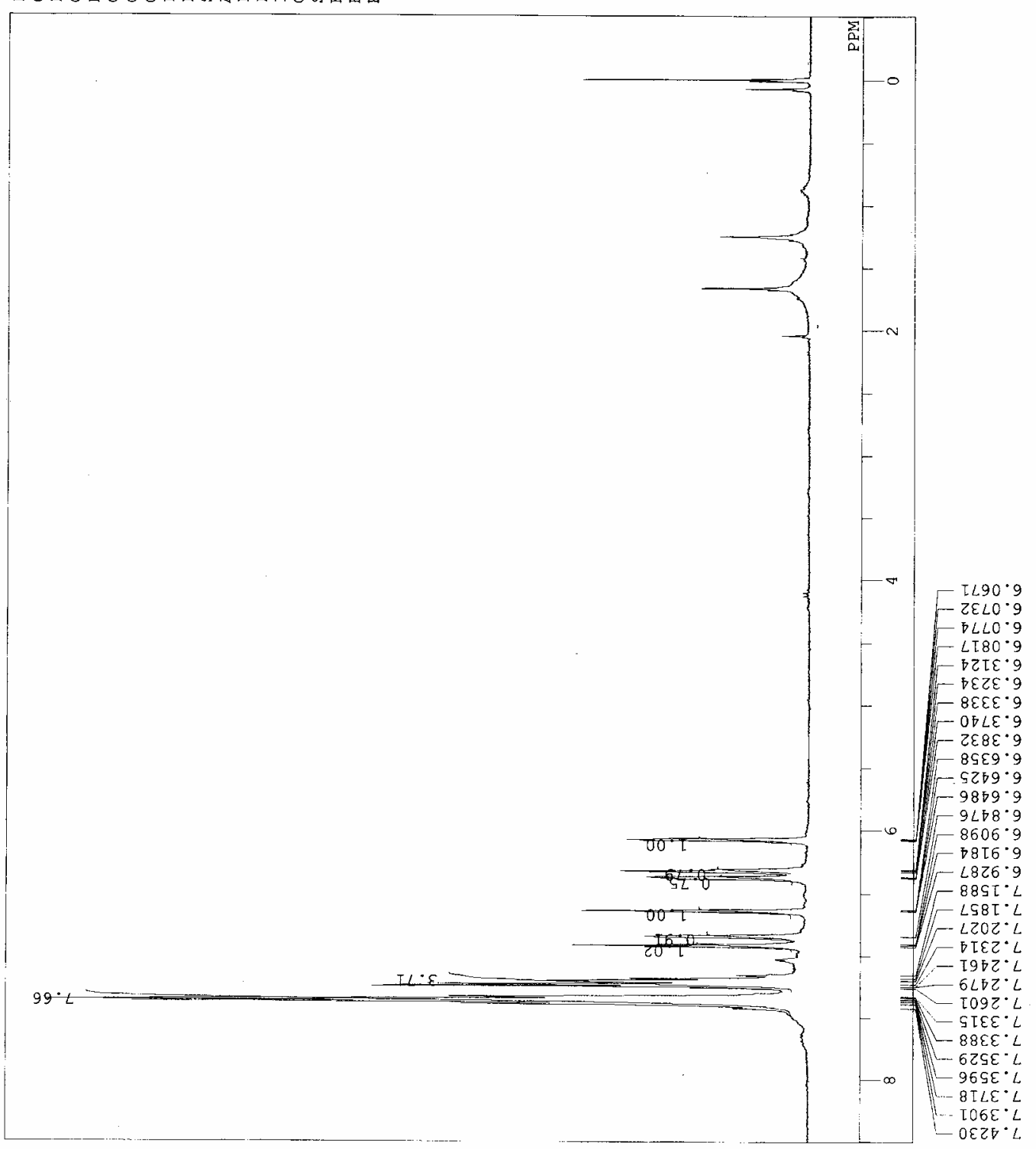




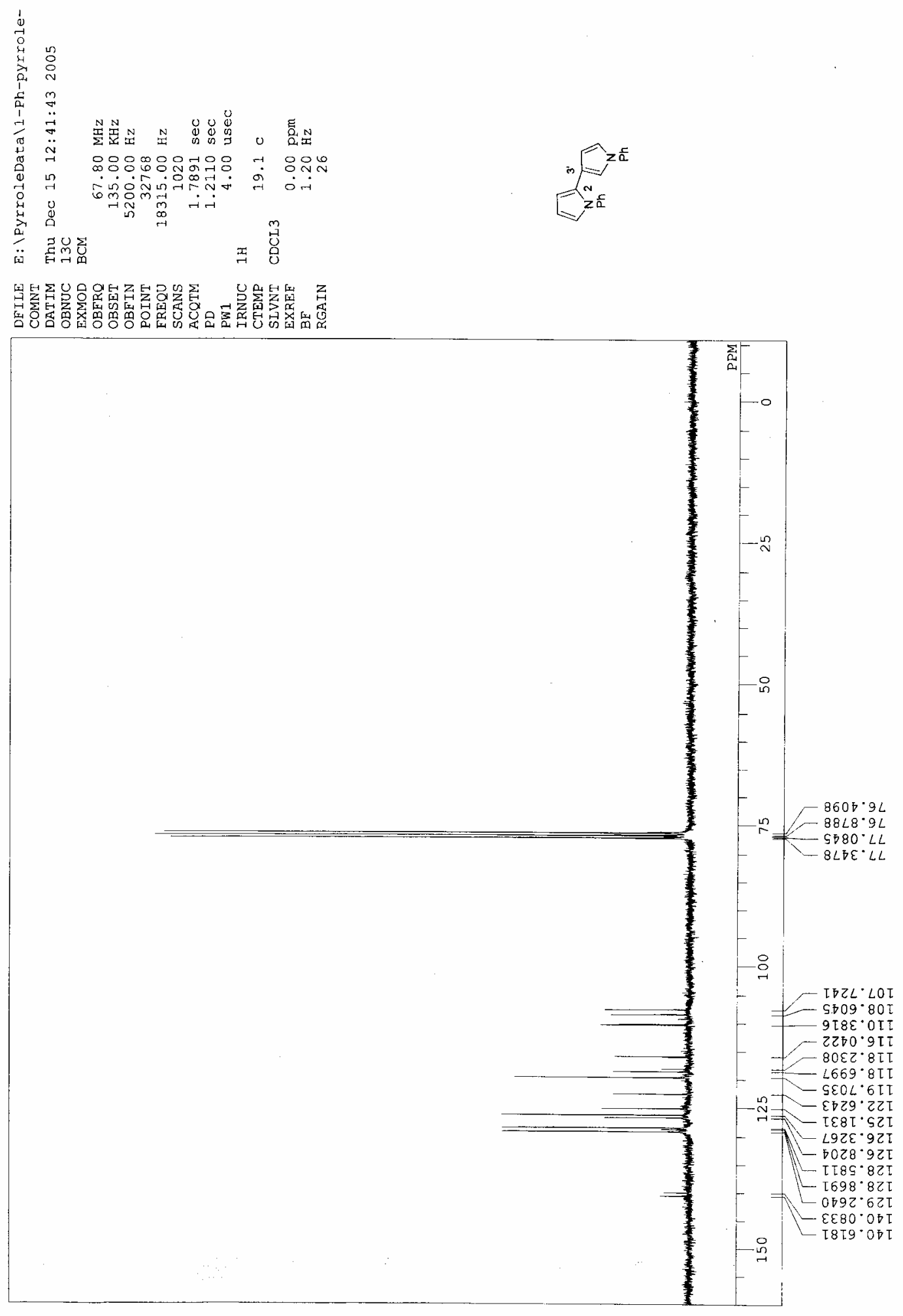

